Alexander Kocks

\title{
Die Theorie der globalen öffentlichen Güter
}

\author{
Forschungsstand und Perspektiven
}

In der Debatte über Global Governance ist der Begriff der globalen öffentlichen Güter inzwischen allgegenwärtig. Doch so inflationär er verwendet wird, so selten findet eine fundierte und systematische Auseinandersetzung mit seinen theoretischen Prämissen und Implikationen statt. Der vorliegende Beitrag nimmt eine Standortbestimmung der aktuellen Forschung über globale öffentliche Güter vor und zeigt dabei auf, dass die Bereitstellungsprognose für diese Güter keineswegs so pessimistisch ausfallen muss wie lange Zeit angenommen. Vielmehr hängt die Frage, wann governance-Akteure bereit sind, einen Beitrag zu diesen Gütern zu leisten, von einer Vielzahl verschiedener Merkmale der sozialen Bereitstellungssituation ab. Dazu gehören nicht nur die nachfrage-, sondern auch die produktionsseitigen Eigenschaften der Güter selbst, Akteurs- und Gruppenmerkmale sowie Bedingungen der rechtlichen, institutionellen und sozialen Umwelt. Je nachdem welche dieser Merkmale vorliegen, ergeben sich völlig unterschiedliche Chancen für die Bereitstellung globaler öffentlicher Güter.

\section{Einleitung ${ }^{1}$}

In der Debatte über Global Governance ist der Begriff der globalen öffentlichen Güter (global public goods) inzwischen allgegenwärtig. Es gibt kaum eine Analyse des Weltregierens durch, mit und ohne den Staat, welche den Begriff nicht zumindest verwendet oder gar durch seine zugrunde liegenden theoretischen Annahmen angeleitet ist. Wann immer wir über die Grenzen der kooperativen Bearbeitung »transsouveräner Probleme« (Rittberger et al. 2009: 346) sprechen, mithin über ineffektives und illegitimes grenzüberschreitendes Regieren, haben wir das governance-Versagen bei der Versorgung mit globalen öffentlichen Gütern im Blick. Für gewöhnlich wird die Unterbereitstellung dieser Güter, deren Nutzen oder Schaden grenzüberschreitend über die regionale Ebene hinaus bis zur globalen Ebene reicht, zurückgeführt auf ihre definierenden nachfrageseitigen Eigenschaften der Nicht-Rivalität im Konsum (die individuelle Nutzung des Guts mindert nicht dessen Verfügbarkeit für andere) und der Nicht-Ausschließbarkeit vom Konsum (niemand kann von der Nutzung des Guts

1 Für hilfreiche Kritik an früheren Fassungen dieses Beitrages danke ich Tine Hanrieder, Markus Kink, Andreas Kruck, Bernhard Zangl und den Teilnehmerinnen und Teilnehmern des IB-Kolloquiums am Geschwister-Scholl-Institut für Politikwissenschaft sowie den drei anonymen Gutachterinnen und Gutachtern und der Redaktion der Zeitschrift für Internationale Beziehungen. 
ausgeschlossen werden). ${ }^{2}$ Ein Beispiel hierfür sind die durch Friedensoperationen generierten öffentlichen Nutzen: Unter der Maßgabe einer erfolgreichen Durchführung können diese Operationen zu globaler Sicherheit und Weltfrieden beitragen, die allen Ländern nutzen - Unterstützern und Nicht-Unterstützern. Von diesen Nutzen kann kein Land ausgeschlossen werden. Zugleich sind globale Sicherheit und Weltfrieden nicht-rivalisierend im Konsum, weil der Gewinn, den ein Land aus ihnen zieht, nicht den Gewinn anderer Länder beeinträchtigt (vgl. Kocks 2007; Shimizu/Sandler 2002).

Spätestens seitdem sich die IB-Forschung - und hier insbesondere die Regimetheorie - der Erkenntnisse zentraler Werke der klassischen Theorie öffentlicher Güter wie »The Logic of Collective Action« (Olson 1965) und »The Tragedy of the Commons« (Hardin 1968) bedient hat, wissen wir, dass öffentliche Güter aufgrund dieser Eigenschaften das Problem des Trittbrettfahrens (free-riding) aufwerfen und deshalb Gegenstand suboptimaler Bereitstellung sind. Warum sollten rationale governanceAkteure einen Beitrag zu globalen öffentlichen Gütern wie Frieden, einer intakten Umwelt, Finanzmarktstabilität, der Kontrolle ansteckender Krankheiten und der Terrorismusabwehr leisten, wenn sie auch ohne Unterstützungsleistung in den Genuss dieser Güter kommen? Es wird angenommen, dass sie ihre wahren Präferenzen für diese Güter nicht bekunden und warten, bis andere sie möglicherweise bereitstellen. Dies hat entweder zur Folge, dass überhaupt kein Mitglied des Kollektivs zur Produktion des Guts beiträgt und dementsprechend auch keine Bereitstellung stattfindet oder, dass zwar Beiträge geleistet werden, jedoch in deutlich geringerem Umfang, als notwendig wäre, um eine kollektiv rationale Lösung zu realisieren (Bobrow/Boyer 2005: 20). Bei öffentlichen Gütern besteht also ein direkter Widerspruch zwischen individuellem Rationalverhalten und Pareto-Effizienz, der sich spieltheoretisch in der strategischen Konstellation des Gefangenendilemmas widerspiegelt. ${ }^{3}$ Die dominante Strategie des einzelnen Akteurs ist es, keinen Beitrag zur Produktion des öffentlichen Guts zu leisten, sodass das Kollektiv ein nur suboptimales Ergebnis erlangen kann (Hardin 1982: 22-30). ${ }^{4}$ Da auf globaler Ebene kein Pendant zum Staat existiert, der als zentrale Lösung der öffentlichen Güterproblematik gesehen wird, stellt sich die

2 Die Definition öffentlicher Güter anhand der Kriterien der Nicht-Rivalität und der NichtAusschließbarkeit stammt von Samuelson (1954). Der Begriffsteil global wird in der Literatur uneinheitlich verwendet. Während einige Autorinnen und Autoren öffentliche Güter erst dann als global bezeichnen, wenn deren positive oder negative Externalitäten tatsächlich umfassend global spürbar sind, folge ich hier den Definitionen, die öffentliche Güter bereits dann als global bezeichnen, wenn sich ihr Nutzen oder Schaden über mehr als nur eine regionale Gruppe von Ländern erstreckt (z.B. Kaul et al. 2003b: 23). Synonym werden in der Literatur hierfür auch die Begriffe internationale öffentliche Güter (Ferroni/Mody 2002) und transnationale öffentliche Güter (Sandler 2004) verwendet. Zudem subsumiere ich unter den Begriff der öffentlichen Güter auch sämtliche unreinen und Sonderformen öffentlicher Güter (siehe unten), während einige Autorinnen und Autoren dafür die Oberbegriffe common goods (Holzinger 2008) oder Kollektivgüter (Rittberger et al. 2009) verwenden.

3 Ein Zustand ist pareto-effizient beziehungsweise pareto-optimal, wenn sich kein Individuum besser stellen kann, ohne zugleich ein anderes Individuum schlechter zu stellen.

4 Eine dominante Strategie ist unter allen möglichen Strategien jene, die das individuell beste Ergebnis liefert, unabhängig davon, wie sich die anderen Akteure entscheiden. 
Prognose für die Bereitstellung globaler öffentlicher Güter unter der Bedingung der Anarchie umso pessimistischer dar. ${ }^{5}$

Aus der aktuellen Literatur zu globalen öffentlichen Gütern wird jedoch deutlich, dass ihre Bereitstellung keineswegs immer durch eine Gefangenendilemmasituation gekennzeichnet ist und auch bei Abwesenheit einer zentralen Steuerungsinstanz substanzielle Beiträge zu diesen Gütern beobachtbar sind. Die Eindämmung der Ausbreitung von Pocken- und Polio-Epidemien, das internationale Postwesen, die globale Koordinierung der Zivilluftfahrt, einheitliche Bankcodes und -standards sowie der multilaterale Abbau von Handelsgrenzen sind nur einige Beispiele für erfolgreich bereitgestellte globale öffentliche Güter, von deren grenzüberschreitendem Nutzen niemand ausgeschlossen werden kann und bei denen keine Rivalität im Konsum herrscht.

Wie lässt es sich aber erklären, dass bei einigen globalen öffentlichen Gütern interund transnationale Kooperation misslingt, bei anderen hingegen Lösungen erreicht werden? Warum versagen governance-Akteure beispielsweise bei der Begrenzung von klimaschädlichen Treibhausgasemissionen, während sie sich auf Vorkehrungen zur Flughafensicherheit im Rahmen der internationalen Terrorismusbekämpfung einigen konnten?

Der ursprünglich prognostizierte Zusammenhang zwischen den definierenden Eigenschaften öffentlicher Güter (Nicht-Ausschließbarkeit und Nicht-Rivalität) und der Wahrscheinlichkeit kollektiven Handelns im Sinne ihrer pareto-effizienten Bereitstellung kann diese Varianzen jedenfalls nicht hinreichend erklären. Vielmehr wird heute eine Vielzahl verschiedener ineinandergreifender Merkmale der Bereitstellungssituation globaler öffentlicher Güter für die Kooperationswahrscheinlichkeit verantwortlich gemacht: Dies sind neben den nachfrage- auch die produktionsseitigen Eigenschaften der Güter selbst, Akteurs- und Gruppenmerkmale sowie Bedingungen der rechtlichen, institutionellen und sozialen Umwelt, unter denen globale öffentliche Güter bereitgestellt werden. Je nachdem, welche dieser Merkmale vorliegen, ergeben sich unterschiedliche Typen strategischer Konstellationen und damit auch ganz unterschiedliche Prognosen für die Bereitstellung globaler öffentlicher Güter (Holzinger 2008; Sandler 2004).

Mit der systematischen Untersuchung dieser verschiedenen Merkmale reicht die Theorie der globalen öffentlichen Güter in ihrer gegenwärtigen Weiterentwicklung weit über ihre ursprüngliche wohlfahrtsökonomische Fundierung und konzeptionelle Verwendung im Rahmen der Regimetheorie hinaus. Eine Bestandsaufnahme der ak-

5 Jedoch hat die public-choice-Theorie gezeigt, dass es auch zum Staatsversagen bei der Bereitstellung öffentlicher Güter auf nationaler Ebene kommen kann (vgl. Aretz 2005). Überdies konnten die Arbeiten von Ostrom (1990) und Ostrom et al. (1994) Bedingungen für eine effiziente Bereitstellung lokaler Gemeinschaftsgüter durch private Akteure aufzeigen, was der für lange Zeit vorherrschenden ökonomischen Einschätzung widerspricht, dass nur der Staat die Bereitstellung öffentlicher Güter sicherstellen kann. Inzwischen werden die Möglichkeiten und Grenzen der privaten Bereitstellung öffentlicher Güter in der ökonomischen Literatur ausgiebig diskutiert. Neben den in diesem Literaturbericht vorgestellten Forschungsarbeiten siehe hierzu z.B. Buttkereit (2009); Besley/Ghatak (2006); Smitha et al. (1995); Bergstrom et al. (1986). 
tuellen wissenschaftlichen Debatte über globale öffentliche Güter scheint daher angebracht. Der vorliegende Beitrag geht der Frage nach, welcher Erkenntniszugewinn sich für die Disziplin der Internationalen Beziehungen ergibt, wenn globale Politikphänomene durch die Brille der globalen öffentlichen Güter betrachtet und untersucht werden. Die Konzentration auf Studien, die explizit zur (Weiter-)Entwicklung einer positiven Theorie der globalen öffentlichen Güter beigetragen und den aktuellen wissenschaftlichen Diskurs über diese Güter entscheidend (mit-)geprägt haben, begründet die Literaturauswahl: ${ }^{6}$

- Barrett, Scott 2007: Why Cooperate? The Incentive to Supply Global Public Goods, Oxford.

- Holzinger, Katharina 2008: Transnational Common Goods: Strategic Constellations, Collective Action Problems, and Multi-Level Provision, Basingstoke.

- Kaul, Inge/Conceição, Pedro/Le Goulven, Katell/Mendoza, Ronald U. (Hrsg.) 2003: Providing Global Public Goods: Managing Globalization, Oxford.

- Sandler, Todd 2004: Global Collective Action, Cambridge, MA.

Ziel des Literaturberichts ist es, sowohl einen Überblick über den Stand der Entwicklung der Theorie der globalen öffentlichen Güter zu geben, als auch ihren konkreten analytischen Mehrwert anhand von vier Leitfragen an diesen Forschungsarbeiten aufzuzeigen:

- Welche Merkmale der Bereitstellungssituation führen zu welchen Kollektivhandlungsproblemen?

- Welche Lösungsvorschläge machen die Studien für diese Kollektivhandlungsprobleme?

- Welche generellen Defizite weisen die Forschungsarbeiten auf?

- Welche zukünftigen Entwicklungslinien lassen sich identifizieren?

Mit Blick auf die theoretischen Annahmen und empirischen Plausibilisierungen der Forschungsarbeiten rekonstruiere ich nachfolgend (siehe Abschnitt 2), welche Merkmale der Bereitstellungssituation den Autorinnen und Autoren zufolge einen Einfluss auf die Kooperationswahrscheinlichkeit bei der Bereitstellung globaler öffentlicher Güter haben. In Abschnitt 3 zeige ich auf, welche generalisierbaren Empfehlungen sich aus den Forschungsergebnissen für die Lösung der Kollektivhandlungsprobleme und damit für die Bereitstellungsoptimierung ergeben. Abschließend (siehe Abschnitt 4) mache ich auf einige Defizite in der Forschung über globale öffentliche Güter aufmerksam und skizziere, wie die Theorie weiterentwickelt werden kann.

6 Einzig der Sammelband von Kaul et al. (2003a) bewegt sich an der Schnittstelle zwischen einer empirisch-analytischen und einer normativ-programmatischen Perspektive, die sich explizit dem normativen Leitbild einer gerechten Weltordnung verpflichtet fühlt und aufzuzeigen versucht, welche Güter wie zur Verfügung gestellt und verteilt werden sollten, um dieses Leitbild zu erreichen. Für den vorliegenden Literaturbericht sind ausschließlich die analytischen Beiträge des Sammelbandes relevant. Zum von Kaul et al. (1999; 2003a) angestoßenen programmatischen Diskurs über globale öffentliche Güter siehe z.B. Carbone (2007); International Task Force on Global Public Goods (2006). 


\section{Bereitstellungsmerkmale und resultierende Kollektivhandlungsprobleme}

Die Annahme, dass nicht nur die nachfrageseitigen Eigenschaften öffentlicher Güter einen Einfluss auf deren Bereitstellungswahrscheinlichkeit und -umfang haben, sondern dass öffentliche Güter in unterschiedlichen sozialen Kontexten bereitgestellt werden, deren spezifische Merkmale die Kooperationsneigung der Akteure beeinflussen, konnte bereits durch einzelne Studien untermauert werden. Dies gilt sowohl für den Einfluss einer Reihe von Eigenschaften der Güter selbst (vgl. Hirshleifer 1983; Cornes/Sandler 1996), den Einfluss von Akteurs- und Gruppeneigenschaften (vgl. Hausken/Plümper 1999; Isaac et al. 1994) als auch für den Einfluss der institutionellen und rechtlichen Umwelt (vgl. Ostrom 1990; Ostrom et al. 1994). Mit expliziter Bezugnahme auf globale öffentliche Güter stand eine systematische theoretische und empirische Klärung, wie die verschiedenen Merkmale die strategische Konstellation beeinflussen und die Wahrscheinlichkeit der Bereitstellung dieser Güter bestimmen, jedoch bis dato aus (vgl. Aggarwal/Dupont 1999: 393; Holzinger 2002: 62).

Es ist diese Syntheseleistung, welche die hier betrachteten Studien auszeichnet. Auch ist ihnen gemeinsam, dass sie Überblicksarbeiten darstellen, in denen die Autorinnen und Autoren ihre langjährigen Forschungsarbeiten zusammenführen und auf eine modellhafte ökonomische Darstellung weitgehend verzichtend - für die Internationalen Beziehungen (IB) fruchtbar machen. Wie alle rationalistischen (IB-)Theorien stellt auch die Theorie der globalen öffentlichen Güter dabei einen strukturell-individualistischen Erklärungsansatz dar, der einen Mehrebenenzusammenhang zwischen der Makro- und der Mikroebene herstellt (Coleman 1991: 27-32). Die kollektiven Explananda (Kooperation mit dem Ziel der Bereitstellung globaler öffentlicher Güter) bilden die Makroebene und werden als ein Effekt des Akteurshandelns auf der Mikroebene interpretiert (Nutzenmaximierung). Das Handeln der einzelnen Akteure wird wiederum durch materielle und soziale Strukturen und Handlungsrestriktionen auf der Makroebene beeinflusst (z. B. Eigenschaften der Güter). Die Verbindung zwischen den auf der Makroebene relevanten Handlungsbedingungen und der Mikroebene erfolgt über entsprechende Brückenannahmen.

Die Vielzahl von Erklärungsvariablen auf der Makroebene, denen die Autorinnen und Autoren dieser Studien einen Einfluss zusprechen, lassen sich dabei einer der oben genannten drei Dimensionen zuordnen: Bei den Merkmalen der Güter sind dies nicht nur die nachfrage- und produktionsseitigen Eigenschaften der bereitzustellenden Güter, sondern auch die geografische Reichweite ihrer Externalitäten. Auf der Ebene der Gruppenmerkmale sind mindestens zu nennen: Gruppengröße, Homogenität vs. Heterogenität der Akteurspräferenzen, Interaktionsdauer und Anonymität vs. Nicht-Anonymität der Akteure. Auf der Ebene der rechtlichen, institutionellen und sozialen Umwelt sind zudem Variablen wie Eigentumsrechte und andere rechtliche Beschränkungen, Kommunikationsmöglichkeit der Akteure, Sanktionsmöglichkeiten und Transparenz zu verordnen. ${ }^{7}$ Angesichts dieses in den Studien vorzufindenden

7 Für eine systematische Zuordnung der verschiedenen Variablen zu diesen Dimensionen siehe auch Holzinger (2008: 36-41; 2002: 63). 
Variablen-Kosmos liegt zunächst der Verdacht nahe, dass die Stärke der Theorie der globalen öffentlichen Güter zugleich ihre Schwäche sein könnte. Zum einen erhebt sie den Anspruch, keine holzschnittartigen Vereinfachungen vorzunehmen, um der realweltlichen Komplexität des globalen Regierens gerecht zu werden. Auf der anderen Seite ist anzunehmen, dass kaum noch originelle Vorhersagen möglich sind, wenn man die Theorie in ihrer gesamten Komplexität in Anschlag bringen würde. Wie Holzinger jedoch zu Recht konstatiert, steht den unendlichen Kombinationsmöglichkeiten der verschiedenen Bereitstellungsmerkmale eine begrenzte Zahl möglicher strategischer Grundkonstellationen gegenüber, die wiederum mit bestimmten Bereitstellungsprognosen einhergehen:

»Die systematische Variation einiger wesentlicher Eigenschaften der Güter (Kosten-Nutzen-Relationen, Rivalität und Ausschließbarkeit, Aggregationstechnologien) und der Gruppen (Homo- und Heterogenität), wobei die anderen Eigenschaften jeweils konstant gehalten werden, zeigt, dass immer wieder die gleichen strategischen Konstellationen entstehen« (Holzinger 2003: 6).

Einige dieser Variablen, die von den Autorinnen und Autoren als unverzichtbare Kernbestandteile der Erklärung von Kooperation bei der Bereitstellung globaler öffentlicher Güter betrachtet werden, sollen nachfolgend genauer beleuchtet werden. Dabei erfolgt die Darlegung der zentralen Prognosen in direkter Verknüpfung mit ausgewählten empirischen Fallbeispielen und -studien aus den hier diskutierten Beiträgen, um der Erklärungskraft dieser Variablen nachzugehen.

\subsection{Nachfrage- und produktionsseitige Gütereigenschaften}

\subsubsection{Nachfrageseitige Eigenschaften: verschiedene Öffentlichkeitsgrade globaler Güter}

Einen wesentlichen Einfluss auf die strategische Akteurskonstellation und damit die Wahrscheinlichkeit des kollektiven Handelns bei der Bereitstellung globaler öffentlicher Güter sprechen die Autorinnen und Autoren der hier ausgewählten Literatur zunächst den nachfrageseitigen Eigenschaften der Güter selbst zu. Der Grundgedanke, dass nicht (nur) die Beschaffenheit des internationalen Systems und die Akteursmerkmale einen Einfluss auf das kollektive Handeln haben, sondern (auch) »the nature of the issue-areas themselves (or the issues they are composed of) « (Hasenclever et al. 1997: 59-60) ist ein Gedanke, der auch dem problemstrukturellen Ansatz innerhalb der Regimetheorie zugrunde lag (vgl. Efinger et al. 1988: 84-116). Eine Differenzierung nach verschiedenen Öffentlichkeitsgraden des jeweiligen Problems wird innerhalb dieses Ansatzes jedoch nicht vorgenommen. ${ }^{8}$

8 Zwar wird auch innerhalb des problemstrukturellen Ansatzes zwischen den Eigenschaften verschiedener bereitzustellender Güter unterschieden, jedoch erfolgt diese Unterscheidung weder nach den Kriterien der Nicht-Auschließbarkeit und Nicht-Rivalität auf der Nachfrageseite noch nach verschiedenen Aggregationstechnologien (siehe Abschnitt 2.1.2) auf der Produktionsseite. 
Hingegen werden von der Theorie der globalen öffentlichen Güter mit verschiedenen Öffentlichkeitsgraden der Güter ganz spezifische collective-action-Prognosen in Verbindung gebracht. Um die Vielfalt und Verschiedenheit tatsächlich beobachtbarer Güterformen auf globaler Ebene adäquat zu erfassen, greifen die Autorinnen und Autoren dabei neben der Unterteilung in rein öffentliche und rein private Güter auf komplexere Taxonomien zurück (vgl. Barett 2007: 20; Holzinger 2008: 19; Sandler 2004: 58-59). So lassen sich nicht nur lokale, nationale und regionale, sondern auch globale öffentliche Güter weiter ausdifferenzieren in:

- $\quad$ unreine öffentliche Güter, die nur eines der beiden Öffentlichkeitskriterien erfüllen (Klubgüter, Allmendegüter);

- Güter, die durch gleichzeitiges und zumeist komplementäres Auftreten öffentlicher und privater Nutzen gekennzeichnet sind (joint product public goods);

- Netzwerkgüter (deren öffentlicher Nutzen sich mit steigendem Anteil an Nutzern vergrößert) und;

- teilweise rivalisierende Güter bzw. congestible goods (deren öffentlicher Nutzen sich mit steigendem Anteil an Nutzern verringert).

Mit jeder dieser nachfrageseitigen Eigenschaften eines Guts werden wiederum unterschiedliche strategische Konstellationen und Erwartungen über seine Bereitstellungswahrscheinlichkeit verknüpft. ${ }^{9}$

So wird beispielsweise wie bei rein öffentlichen Gütern auch für Allmendegüter (kein Ausschluss, aber Rivalität im Konsum) die strategische Konstellation des Gefangendilemmas prognostiziert. Ein Standardbeispiel für ein Allmendegut sind die Fischbestände im offenen Meer: Es besteht kein Ausschluss vom Fischfang, aber Rivalität, da jeder Fisch nur einmal gefangen werden kann. Es ist zwar im Interesse aller Fischer, insgesamt nur soviel zu fangen, dass die Art erhalten bleibt, aber bei ausschließlich individueller Entscheidung über die jeweiligen Fangmengen ist eine Überfischung wahrscheinlich. Die Gefangenendilemmasituation ist offensichtlich: Jeder Fischer steht vor der Wahl, die Netze vollständig zu füllen oder zur Arterhaltung beizutragen. Schränken sich die anderen Fischer nicht ein, dürfte die Überfischung unvermeidbar sein - auch wenn der Einzelne auf den Fang verzichtet. In diesem Fall ist es für ihn die beste Strategie, ebenfalls so viel wie möglich zu fangen. Sichern die anderen Fischer hingegen die Arterhaltung, so wäre es für den Einzelnen erst recht lukrativ, die Netze zu füllen. Wie auch immer sich die anderen Fischer verhalten, für den Einzelnen ist die dominante Strategie, die Fangmenge zu maximieren (Sandler 2004: 55-60; Weinmann 2001: 132-133). Das Gefangenendilemma führt somit zu einer Ausbeutung der global commons. Allerdings konnten die Arbeiten von Ostrom (1990) und Ostrom et al. (1994) durchaus Bedingungen aufzeigen, unter denen es bei verschiedenen lokalen Allmendegütern zu erfolgreicher Institutionalisierung und kooperativer Selbstorganisation kommen kann.

9 Nachfolgend werden nicht alle hier genannten Güterarten und die mit ihnen verbundenen collective action-Prognosen gleichermaßen ausführlich dargestellt. Der Fokus auf einige ausgewählte Beispiele soll dazu dienen, die grundlegende Argumentationslogik der Theorie zu explizieren. 
Bei Klubgütern (keine oder geringe Rivalität im Konsum, aber Konsumausschluss) sind die Bereitstellungsanreize für Klubmitglieder und solche, die es werden wollen, in der Regel stärker als bei reinen öffentlichen Gütern und Allmendegütern. ${ }^{10} \mathrm{Si}$ cherheitsallianzen und Organisationen der Wirtschaftsintegration sind in Bezug auf Mitgliedschaften selektiv und schließen Außenstehende vom Nutzen des Klubguts aus. In Situationen, in denen neue Mitglieder aufgenommen werden, wägen die gegenwärtigen Mitglieder den potenziellen Effekt neuer Mitgliedschaften auf die Bereitstellung des jeweiligen Guts (z.B. NATO-Bündnisverteidigung) ab. Sehen Kandidaten eine potenzielle Mitgliedschaft als gewinnbringend an, so haben sie einen Anreiz, Beiträge zur Güterbereitstellung zu leisten (Bobrow/Boyer 2005: 22). Der Grund für Klubformationen ist, dass ein universeller Zugang zu Überfüllungsphänomenen und somit zu Rivalität führen würde. Durch die Eingrenzung des Zugangs auf beitragszahlende Mitglieder wird hingegen sichergestellt, dass das Gut nur den Klubmitgliedern zur Verfügung steht und free-riding ausgeschlossen werden kann. Mitglieder mit einer größeren Präferenz für das Klubgut werden dieses häufiger nutzen und dabei mehr Gesamtgebühren zahlen. Der Ausschlussmechanismus zwingt die Mitglieder, ihre Präferenzen zu enthüllen, so dass sie den Klub so oft besuchen (respektive für das Gut zahlen), bis die Gebühren den erzielten Nutzen entsprechen (Arce/Sandler 2002: 15). Die erhobenen Gebühren können dann genutzt werden, um die Gesamtbereitstellung sicherzustellen, so dass Klubs einen effizienten Allokationsmechanismus gewährleisten (Sandler 2004: 52-53; Buchanan 1965: 1-14). In einem idealen Klub, in dem die Überfüllungsgebühren genutzt werden, um die monitoringund Bereitstellungskosten des Guts zu internalisieren, liegt daher auch keine problematische strategische Konstellation vor.

Auch für globale joint-product public goods, d.h. Güter, die gleichzeitig sowohl private als auch öffentliche Nutzen beinhalten (Sandler 2004: 53), können sich positive Bereitstellungsszenarien ergeben. Entscheidend ist dabei Sandler zufolge das Verhältnis der jeweiligen Nutzen, so dass - spieltheoretisch betrachtet - unterschiedliche strategische Konstellationen (z. B. das Versicherungsspiel) möglich sind. Kennzeichnend für diese Güterart ist zudem, dass sich die verschiedenen Nutzen gegenseitig verstärken und kollektives Handeln ermöglichen können:

$»$ If the $[. .$.$] contributor-specific benefits are complementary to the purely public benefits$ when contributors desire to consume these derived outputs in conjunction, then increased spillovers of the purely public components may encourage more, rather than less, collective provision. In this case, bundling is important because spillover recipients cannot receive the complementary contributor-specific benefits unless they support the collective action« (Sandler 2004: 55).

Sandler plausibilisert diese Prognose in seiner Fallstudie zu internationalen Peacekeeping-Einsätzen (Sandler 2004: 192-211). Weil sich der Zusammenhang zwischen Gütereigenschaften und Bereitstellungswahrscheinlichkeit mit dieser Fallstudie sehr gut veranschaulichen lässt, soll sie hier ausführlicher dargestellt werden. Sandler zufolge, sehen sich internationale Organisationen wie die UNO, NATO und EU allo-

10 Zur Theorie der Klubgüter und ihrer Anwendung siehe z.B. Cornes/Sandler (1996); Bernauer (1995); Buchanan (1965). 
kativen Effizienzproblemen bei der Bereitstellung von Friedensoperationen gegenüber, wenn diese ausschließlich globale (öffentliche) Nutzen wie beispielsweise Frieden, Sicherheit und Stabilität generieren. Gemessen an ihrer output-Seite würden Friedensoperationen in diesem Fall reine globale öffentliche Güter darstellen. Da Friedensoperationen aber zugleich auch länderspezifische (private) Nutzen wie zum Beispiel Reputationsgewinne, höhere Rückerstattung für bereitgestellte Truppen und die Verwirklichung ökonomischer Interessen in der Konfliktregion generieren können, stellen sie nach Sandler globale joint product public goods dar. Sandlers Ansatz, der unmittelbar an Olsons Nebenprodukttheorie (Olson 1965: 132-168) anknüpft, stellt eine Generalisierung von beidem, dem reinen öffentlichen und dem privaten Gütermodell, dar und beinhaltet jedes als spezifischen Fall. Dementsprechend prognostiziert Sandler, dass die Unterstützung von Friedensoperationen vom Anteil der privaten Nutzen an den Gesamtnutzen (Summe aus privaten und öffentlichen Nutzen) abhängt. Je höher dieser Anteil für einzelne Staaten ist, desto mehr antizipieren sie die Unterstützung dieser Operationen als attraktivste Handlungsalternative. Denn sie würden die privaten Nutzen nicht erhalten, solange sie sich nicht an der Unterstützung der Operation beteiligen (Sandler 2004: 204). Trittbrettfahren wäre in diesem Fall also nicht rational. Würden Friedensoperationen hingegen ausschließlich rein öffentliche Nutzen generieren, wären sie dem Ansatz zufolge Gegenstand suboptimaler Allokation und damit von Unterbereitstellung. Die Suboptimalität ihrer Bereitstellung nähme in dem Maße zu, wie für die globalen benefit spillovers von den Trittbrettfahrern kein Preis entrichtet wird, mithin also keine Internalisierung der Nutzen vollzogen wird.

Um seinen Ansatz zu testen, führt Sandler eine Reihe quantitativer Analysen durch, die offenlegen sollen, ob die Lasten für Friedensoperationen unter den Mitgliedstaaten proportional oder disproportional getragen werden. Disproportionalität wird dabei als Ausdruck des überwiegenden Vorhandenseins öffentlicher Nutzen interpretiert - eine Annahme, die in unmittelbarem Zusammenhang zur Variable der Gruppengröße (siehe Abschnitt 2.2) steht und sich bereits bei Olson (1965) und Olson und Zeckhauser (1966) findet. Ihnen zufolge besteht in kleinen privilegierten Gruppen zwar anders als in großen Gruppen weniger das Problem, sich überhaupt mit reinen öffentlichen Gütern zu versorgen, jedoch eine starke Tendenz zur suboptimalen (pareto-ineffizienten) Versorgung mit diesen Gütern, die sich als eine Ausbeutung der Großen durch die Kleinen (Olson 1965: 29) darstellt. Mit den Großen sind dabei jene gemeint, die bereit sind, die Kosten für das Gut alleine zu tragen. Da sie über die nötigen Ressourcen verfügen, können sie die mit der Güterbereitstellung verbundenen Opportunitätskosten eher in Kauf nehmen als ressourcenärmere Akteure. ${ }^{11}$ Dieser Gedanke wurde auch von der Theorie hegemonialer Stabilität (Kindleberger 1981; Gilpin 1987) aufgegriffen.

11 Dies kann selbst bei homogener Nutzenverteilung gelten, umso mehr aber bei heterogener Nutzenverteilung, die einen noch höheren Anreiz zur uni- oder minilateralen Bereitstellung schafft (siehe Abschnitt 2.2). 
Um das Lastenteilungsverhalten bei der Finanzierung von Friedenseinsätzen zu überprüfen, hat Sandler für den Zeitraum 1976-1991 die tatsächlich geleisteten Zahlungen jener 29 UN-Mitgliedstaaten, die nach dem damals gültigen Beitragsschlüssel nahezu den gesamten Haushalt für UN-Peacekeeping-Operationen aufzubringen hatten, erfasst und in Beziehung zum Bruttoinlandsprodukt dieser Länder gesetzt. Mittels Korrelationsanalysen weist er nach, dass die Variablen Bruttoinlandsprodukt und tatsächlich geleistete Zahlungen nicht positiv miteinander korrelieren. Daraus könne geschlossen werden, dass während des Kalten Krieges entsandte Operationen vornehmlich den privaten Interessen einzelner Blockländer dienten (Sandler 2004: 205-209; Khanna et al. 1998; 1999). Hingegen konnte für den Zeitraum 1994-2000 eine positive Korrelation der beiden Variablen nachgewiesen werden (Shimizu/Sandler 2002). Dieser Zusammenhang bestätige die von Olson und Zeckhauser (1966) postulierte Ausbeutungshypothese: Das free-riding der kleinen Mitgliedsstaaten spräche für das Überwiegen rein öffentlicher Nutzen. In anderen Worten: Nach dem Ende des Kalten Krieges ist die rein öffentliche Güter-Komponente bei UN-Friedensoperationen als globalen joint-product public goods dominanter als ihre private Komponente. Ferner habe sich das Verhältnis seit den 1990er Jahren stetig zugunsten der öffentlichen Nutzen verschoben (Sandler 2004: 205-209).

Auch wenn Sandlers Vorgehensweise grundsätzlich darauf abzielt, den Einfluss nachfrageseitiger Eigenschaften von Friedenseinsätzen auf die Kooperationsgeneigtheit der Akteure bei Bereitstellung dieses Gutes zu plausibilisieren, ist sie kritikwürdig, da sie keinen echten empirischen Test darstellt. Die Grundannahme seiner theoretischen Überlegungen - nämlich, dass disproportionale Lastenteilung und die daraus resultierenden Bereitstellungsdefizite Ausdruck eines überwiegenden Vorhandenseins öffentlicher Nutzen sind - wird in tautologischer Weise vorausgesetzt. Die Nutzenrelation (unabhängige Variable) wird schlichtweg aus dem theoretisch prognostizierten Verhalten der Lastenteilung (abhängige Variable) post hoc abgeleitet, ohne die Ausprägung der unabhängigen Variable zu messen. In einem empirischen Test müsste jedoch der Versuch unternommen werden, die Erwartungsnutzen der Akteure und die daraus resultierenden Nutzenrelationen unabhängig von dem Ergebnis, das sie erklären sollen, zu erheben. ${ }^{12}$ Demnach wäre das Verhältnis von zu erwartenden privaten Nutzen und dem Gesamtnutzen direkt zu erfassen, um zu prüfen, wie sich diese Varianzen in entsprechende Varianzen der Akteurskooperation übersetzen. Im direkten Vergleich der Unterstützung einzelner Operationen könnte sich dann durchaus zeigen, dass zum Beispiel die KFOR-Operation im Kosovo eben deshalb stärker finanziell, personell und materiell unterstützt wurde, als zum Beispiel die ISAF-Mission in Afghanistan, weil sie mehr private Nutzen für die Unterstützer in Aussicht gestellt hatte (vgl. Kocks 2009).

$12 \mathrm{Zu}$ den Vor- und Nachteilen einer direkten Nutzen- und Präferenzermittlung siehe z.B. Bienen et al. (1999: 10-11); Hasenclever et al. (1997: 24-25); Zürn (1997; 1992); Snidal (1985a). 


\subsubsection{Produktionsseitige Eigenschaften: Aggregationstechnologien}

Neben der Unterscheidung zwischen Gütern nach ihren nachfrageseitigen Eigenschaften differenzieren die Autorinnen und Autoren globale öffentliche Güter zudem hinsichtlich ihrer produktionsseitigen Eigenschaften bzw. ihrer »Aggregationstechnologie « (Hirshleifer 1983), welche die Art und Weise beschreibt, mit der die individuellen Beiträge zu einem globalen öffentlichen Gut seinen Gesamtumfang festlegen. Die Studien unterscheiden fünf verschiedene Arten dieser »third property of publicness« (Sandler 2004: 60), die von Gut zu Gut variieren können und mit denen wiederum unterschiedliche strategische Akteurskonstellationen und Bereitstellungsprognosen in Verbindung gebracht werden. So wird prognostiziert, dass kollektives Handeln im Sinne einer effizienten Bereitstellung am unwahrscheinlichsten im Falle von summation-Gütern sei, bei denen sich die Beiträge aller Akteure additiv zur Gesamthöhe aggregieren (z.B. Produktion von Treibhausgasen). Insbesondere bei dieser Gütervariante, von der auch Olson (1965) implizit ausging, wird die Gefahr von freeriding und die Konstellation des Gefangenendilemmas als besonders hoch eingeschätzt. Hingegen eröffne sich für weakest-link-Güter, bei denen der geringste Beitrag die Gesamthöhe des Guts bestimmt (z.B. Seuchenbekämpfung) und für thresholdGütern, bei denen erst eine bestimmte Beitragschwelle erreicht werden muss, damit ihre Nutzen zum Tragen kommen (z.B. Truppenbereitstellung für Friedenseinsätze), die Konstellation des Versicherungsspiels (Holzinger 2008: 69-82; 2001: 128; Sandler 2004: 63). Für best-shot-Güter, bei denen der höchste bzw. beste Beitrag den Gesamtumfang bestimmt (z.B. das am weitesten fortgeschrittene unpatentierte Wissen zur Heilung von Krankheiten), wird die Konstellation eines Koordinationsspiels vorhergesagt, während für weighted-sum-Güter, bei denen sich die Einzelbeiträge additiv, aber mit unterschiedlichem Gewicht zur Gesamthöhe aggregieren (z.B. Einspeisungen in Energieversorgungsnetze), eine Vielfalt möglicher strategischer Konstellationen prognostiziert wird (Sandler 2004: 68). Nachfolgend werden empirische Plausibilisierungen der collective-action-Prognosen für summation-, weakest-linkund best-shot-Güter als paradigmatische Beispiele dargestellt.

\section{(1) Summation-Güter}

Forschungsarbeiten zur Problematik (globaler) öffentlicher Güter gehen in der Regel davon aus, dass ihre Bereitstellung einer summation-Technologie folgt. Kennzeichnend für summation-Güter ist, dass alle Beiträge zu diesen Gütern perfekte Substitute sind, sodass der Beitrag jedes einzelnen Akteurs im selben Ausmaß zur Gesamthöhe des bereitzustellenden Guts beiträgt und es keine Rolle spielt, wo und von wem diese austauschbaren Beiträge geleistet werden (vgl. Sandler 2004: 61; Holzinger 2001: 
124). ${ }^{13}$ Die Anhäufung von Treibhausgasen ist ein klassisches Beispiel für ein globales öffentliches Gut, das der summation-Technologie folgt. Wenn 200 Staaten jeweils 1000 Tonnen an Treibhausgasen in die Atmosphäre emittieren, resultieren 200.000 Tonnen, die die Atmosphäre erwärmen. Weil jede beigesteuerte Einheit denselben Effekt auf die Gesamthöhe hat, ist jede Einheit ein perfektes Substitut für jede andere Einheit. Genau darin liegt, den hier diskutierten Studien zufolge, der Grund für eine dilemmatische Interaktionsstruktur und die damit verbundene Vorhersage der Unterbereitstellung (globaler) öffentlicher Güter. Leisten andere Akteure keinen Beitrag zu einem globalen öffentlichen summation-Gut, ist es für den einzelnen Akteur rational, ebenfalls keinen Beitrag zu leisten. Aber selbst wenn sich die anderen Akteure entscheiden sollten, mehr Einheiten eines solchen Guts bereitzustellen, hat der Einzelne keinen Anreiz, seine Beiträge ebenfalls zu erhöhen, sondern diese vielmehr als Trittbrettfahrer zu verringern (vgl. Barrett 2007: 6, 101; Sandler 2002: 95-96; 2003: 134-135).

So wird üblicherweise für die summation-Technologie die Situation des Gefangenendilemmas prognostiziert. Holzinger plausibilisiert dies mittels eines einfachen 2x2-Matrix-Spiels am Beispiel des intermediären globalen öffentlichen Guts Verringerung von Treibhausgas-Emissionen (Holzinger 2008: 72-75). ${ }^{14}$ Mit der Reduktion dieser Gase würden die Akteure zum finalen öffentlichen Gut Eindämmung der globalen Erwärmung beitragen. ${ }^{15}$ In Holzingers Beispiel müssen sich zwei Akteure entscheiden, ob sie ihre Treibhausgas-Emissionen um eine Einheit reduzieren oder dies unterlassen. Ihre jeweiligen Reduktionen sind additiv und substituierbar. Wie werden sich die Akteure verhalten, wenn ihre jeweiligen Reduktionseinheiten mit einem Nutzenwert von nur sechs $(\mathrm{n}=6)$, aber einem Kostenwert von acht $(\mathrm{k}=8)$ verbunden sind? ${ }^{16}$ Die dominante Strategie beider Akteure ist es, keinen Beitrag zu leisten, weil ihnen bei alleiniger Betrachtung ihres Beitrages nur Kosten anfallen ( $\mathrm{n}=-2)$, das Trittbrettfahren hingegen einen (öffentlich konsumierbaren) Nutzenwert von sechs einbringt, der sich aus dem Beitrag des anderen Akteurs ergibt. Auch wenn kollektives Handeln jedem Akteur einen Nutzenwert von vier (2n-k) einbringen würde, wählt jeder Akteur die individuell rationale Nash-Gleichgewichtsstrategie des Trittbrettfahrens, sodass kein pareto-optimales Ergebnis (beidseitige Reduktion der Treib-

13 Weil die individuellen Beiträge zu summation-Gütern perfekte Substitute sind, hätte auch eine potentielle Einkommensumverteilung keinen Einfluss auf das aggregierte Bereitstellungsniveau: Die höheren Beiträge des einen Akteurs werden exakt durch die verringerten Beiträge des anderen Akteurs ausgeglichen. Dieser Umstand wird als Neutralitätstheorem bezeichnet (vgl. Sandler 2004: 61-62; Bergstrom et al. 1986).

14 Für den Zweck dieses Literaturberichts wird auf Matrix-Darstellungen verzichtet. Entsprechende Abbildungen kann der interessierte Leser oder die interessierte Leserin an den entsprechenden Stellen der hier zitierten Literatur finden.

15 Intermediäre Güter (z.B. Friedensmissionen, Regime, Institutionen) sind solche Güter, die zur Produktion finaler öffentlicher Güter beitragen und ebenfalls öffentliche (mitunter aber auch private) Eigenschaften aufweisen (vgl. Kaul et al. 2003a: 604).

16 Anders als bei Holzinger sind die jeweiligen pay-offs hier als metrische Werte dargestellt. 
hausgas-Emissionen) erzielt werden kann. ${ }^{17}$ Die individuell beste Antwort, sowohl auf Kooperation als auch auf Nicht-Kooperation, ist für ihn die Nicht-Kooperation. In diesem worst-case-scenario leistet also keiner der Akteure einen Beitrag, mit der Folge, dass das globale öffentliche Gut nicht bereitgestellt werden kann (vgl. Holzinger 2008: 74; Sandler 2002: 97).

Die empirischen Fallstudien von Barrett und Sandler zum Scheitern des Kyotoprotokolls untermauern diese Dilemmasituation (Barrett 2007: 84-102; Sandler 2004: 221-234). Auch sie identifizieren den summation-Charakter der Reduktion von Treibhausgasemissionen als zentrales Problem des globalen Klimaschutzes. Obwohl sich die internationale Staatengemeinschaft im Kyotoprotokoll auf völkerrechtlich verbindliche Reduktionsziele bezüglich des Ausstoßes von Treibhausgasen geeinigt hatte, ist insgesamt ein Anstieg von Treibhausgasen, insbesondere des Kohlenstoffdioxids, zu verzeichnen. Sowohl Barrett als auch Sandler führen dies im Wesentlichen darauf zurück, dass die Kosten pro reduzierter Emissionseinheit von den beteiligten Akteuren höher als deren Nutzen antizipiert werden $(n-k>0)$. Die dominante Strategie der Emittenten ist es, den Ausstoß nicht zu reduzieren. Da für Schwellenländer mit inzwischen sehr stark anwachsenden Emissionsraten (z.B. Südkorea, Indien und Brasilien) aber geringen Pro-Kopf-Emissionen keine Emissionsbeschränkungen im Protokoll festgelegt wurden, waren Sandler zufolge vor allem die USA nicht bereit, das Protokoll zu unterzeichnen, solange das Trittbrettfahren für diese Länder im Protokoll quasi institutionalisiert wurde.

\section{(2) Weakest-link-Güter}

Anders als für summation-Güter prognostizieren die Autorinnen und Autoren der hier untersuchten Studien eine effiziente Bereitstellung für weakest-link-Güter, die sich dadurch auszeichnen, dass ihre Gesamthöhe durch den geringsten Beitrag definiert wird. Beispiele für globale öffentliche Güter, die sich nach dieser Aggregationstechnologie zusammensetzen, sind die vorsorgende Seuchen- und Epidemiebekämpfung (vgl. Barrett 2007: 47-73), die Verhinderung terroristischer Aktivitäten in Form von Flughafenkontrollen (Sandler 2002: 98-99) sowie die Bekämpfung von Steuerflucht in Form der Verhinderung von Steueroasen (Holzinger 2003: 6). Eine effiziente Allokation von Ressourcen ist den Autorinnen und Autoren zufolge bei weakest-linkGütern wahrscheinlich, weil kein Anreiz zum Trittbrettfahren besteht: Würde nur einer der beteiligten Akteure keinen Beitrag leisten, wäre das Gut nicht existent. Vielmehr ist zu erwarten, dass die Akteure ihre Beiträge an den geringsten noch ausreichenden Beitrag (weakest link) angleichen, da jede darüber hinausgehende Leistung eine Verschwendung von Ressourcen darstellen würde, ohne dabei den Umfang des öffentlichen Guts zu steigern (Sandler 2002: 98; 2003: 135). Bereits Jack Hirsh-

17 Ein Nash-Gleichgewicht beziehungsweise Nash-Equilibrium ist eine Strategiekombination, von der ausgehend kein einzelner Akteur einen Vorteil für sich erzielen kann, wenn er einseitig von der Strategie abweicht. 
leifer hatte diese Aggregationslogik am Beispiel des lokalen Überschwemmungsschutzes durch Deichbau verdeutlicht: Haben sich die Bewohnerinnen und Bewohnern einer Insel freiwillig darauf geeinigt, ihren jeweiligen Küstenabschnitt durch Deiche zu sichern, und würde nur ein Bewohner oder eine Bewohnerin entgegen der Vereinbarung keinen Deich bauen, so könnte eine Sturmflut die ganze Insel über diesen fehlenden Deich überfluten. Jeder verweigerte Beitrag oder ein solcher, der unterhalb des geringsten notwendigen Beitrags (hier etwa in Form einer Mindestdeichhöhe) liegt, würde den Umfang des Guts gegen Null tendieren lassen. Zugleich würde ein unnötig höher gebauter Deich lediglich Mehrausgaben verursachen, ohne den Überschwemmungsschutz dadurch verbessern zu können (Hirshleifer 1983: 371-372).

Die Studien von Holzinger (2008) und Sandler (2004; 2003) zeigen spieltheoretisch, dass das Akteurshandeln bei der Bereitstellung von weakest-link-Gütern der strategischen Konstellation des Versicherungsspiels entspricht. Anders als im Gefangenendilemma ist Kooperation hierbei in der Regel selbstdurchsetzend, weil sie die höchsten Auszahlungsgewinne für alle Akteure verspricht. ${ }^{18}$ Während sich Holzinger (2008: 75-80) für den Bereich der Biodiversität interessiert und dort den Schutz eines spezifischen Ökosystems als Versicherungsspiel modelliert, plausibilisiert Sandler (2004: 62-64) die strategische Entscheidungssituation am Beispiel der Terrorismusabwehr in Form von Flughafenkontrollen. Folgt man seinem Beispiel, dann ist ein bestimmtes Maß an effektiver Kontrolle notwendig, um die Terrorismusabwehr in diesem Bereich sicherzustellen. Setzt ein Land dieses Mindestmaß an seinen Flughäfen um, ist wahrscheinlich, dass alle anderen Akteure gleichziehen. Der weakest-link-Charakter erfordert, dass alle Akteure dazu beitragen, ansonsten kann das Gut nicht bereitgestellt werden. In diesem Fall hätte kein Spieler einen Vorteil, wenn er einseitig von der Strategie der Kooperation abweichen würde. Würde nur ein Spieler keinen Beitrag leisten, könnte das Gut der Terrorismusabwehr nicht bereitgestellt werden, und auch der Spieler selbst wäre potenziellen Anschlägen ausgesetzt. Damit wären die Kosten seiner Nicht-Kooperation in Form der Terrorismusgefahr höher als sein möglicher Nutzen in Form alternativer Ressourcenverwendungen. Die übrigen Bereitsteller hätten hingegen nur Kosten (Flughafenkontrollen), aber keinerlei Nutzen, weil das Gut der Terrorismusabwehr trotz ihrer Maßnahmen nicht existent wäre (Sandler 2004: 62-64). In dieser strategischen Konstellation liegen zwei NashGleichgewichte vor: Zum einen die allseitige Nicht-Kooperation und zum anderen die allseitige Kooperation im Sinne der Angleichung an den geringsten ausreichenden Beitrag. Nur im zweiten Fall handelt es sich um ein pareto-optimales Gleichgewicht, und da es zugleich das auszahlungsdominante Gleichgewicht ist (Sicherstellung der

18 Während im Gefangenendilemma jeder Akteur das individuell beste Ergebnis durch NichtKooperation erzielt, erhält er dies im Versicherungsspiel durch Kooperation - allerdings nur unter der Voraussetzung, dass auch der andere kooperiert. Daher liegt dem Versicherungsspiel ein Koordinationsproblem zugrunde. 
Terrorismusabwehr), kann erwartet werden, dass die Akteure genau diese Gleichgewichtsstrategie wählen. ${ }^{19}$

So existiert bei Weakest-Link-Gütern keine dominante Strategie; die beste Strategie des Einen hängt von der Strategie des Anderen ab, und die heißt in jedem Fall Kooperation in Form der Bereitstellung des Guts (vgl. Holzinger 2001: 128). Sollte der geringste Beitrag eines Akteurs gegen Null tendieren, da der Akteur nicht über die nötigen Ressourcen verfügt (z.B. Entwicklungsländer) oder nicht in der Lage ist, eine notwendige Leistung zu organisieren (z.B. failed states), sind Transferleistungen der anderen Akteure sehr wahrscheinlich um die Bereitstellung des Gutes sicherzustellen (vgl. Vicary/Sandler 2002).

\section{(3) Best-shot-Güter}

Anders als bei weakest-link-Gütern wird der Gesamtumfang von best-shot-Gütern nicht durch den geringsten, sondern allein durch den höchsten bzw. besten individuellen Beitrag, den »single best effort« (Barrett 2007: 199), bestimmt. Auch für diese Güter, bei denen ein einziger Beitrag ausreicht, um sie für alle Konsumenten zur Verfügung zu stellen, prognostizieren die Autorinnen und Autoren eine höhere Bereitstellungswahrscheinlichkeit als für summation-Güter. Globale öffentliche Güter, deren Bereitstellung einer best-shot-Technologie entspricht, sind beispielsweise die unilaterale Bereitstellung eines Raketenabwehrsystems als grenzüberschreitendes Klubgut und das ursprünglich vom amerikanischen Militär entwickelte und nun weltweit zur Verfügung stehende Global Positioning System (GPS) (vgl. Barrett 2007: 31-32; Sandler 2004: 66-68). Im Mittelpunkt der empirischen Fallstudien stehen ferner die Generierung neuen Wissens durch Forschung und Entwicklung (R\&D) bei der Erfindung von Medikamenten und Impfstoffen für Krankheiten wie Malaria und Ebola (Sandler 2004: 99-121) sowie die Grundlagenforschung in den Bereichen der nachhaltigen Energiegewinnung und des Klimaschutzes (Barrett 2007: 37-46).

Das Charakteristikum von best-shot-Gütern ist, dass ihre Bereitstellung vom technologischen Durchbruch eines einzelnen Akteurs (bzw. einer Akteursgruppe wie einem Team von Forschern und Forscherinnen) abhängt. Solange er seinen Nutzen aus diesem Gut höher einschätzt als seine dafür aufgewendeten Kosten, wird er das öffentliche Gut produzieren. Durch seinen single best shot ist das Gut dann für alle anderen Akteure verfügbar. Nur sein Beitrag legt fest, ob das Gut bereitgestellt werden kann, und alle geringeren Leistungen der anderen Akteure nehmen keinen Einfluss auf Umfang und Qualität des Guts: »whoever is first to supply the good provides it

19 Im auszahlungsdominanten Gleichgewicht erhalten die Spieler einen größeren Gewinn als in anderen Gleichgewichten. 
for everyone « (Sandler 2004: 66). ${ }^{20}$ Sandler und Barrett zufolge liegt der Bereitstellung von best-shot-Gütern ein Koordinationsspiel mit mehreren Nash-Gleichgewichten zugrunde, in dem die Akteure eine einheitliche Entscheidung treffen müssen. Da in realweltlichen Bereitstellungssituationen in der Regel mehrere best-shot-Kandidaten infrage kommen (z.B. verschiedene Gruppen von Forschern und Forscherinnen, die an der Entwicklung eines Impfstoffes arbeiten), müssen sich die Akteure untereinander koordinieren, wer der best shooter sein soll, um keine Ressourcen zu verschwenden (vgl. Barrett 2007: 33-46; Sandler 2002: 99). Sandler verdeutlicht dies mithilfe eines einfachen 2x2-Spiels: Zwei Akteure müssen sich jeweils entscheiden, ob sie das Gut zu einem öffentlich konsumierbaren Nutzenwert von sechs und einem individuellen Kostenwert von vier bereitstellen. Derjenige Akteur, der den best shot erbringt, erhält einen positiven Nutzenwert von zwei (6-4=2), der Nicht-Bereitsteller hingegen einen positiven Nutzenwert von sechs, weil für ihn keine Kosten anfallen. Wenn aber beide Akteure den best shot leisten würden, wäre der zweite Beitrag redundant: Er würde keinerlei zusätzlichen Nutzen einbringen, weil das Gut bereits existiert, aber unnötige zusätzliche Kosten verursachen, sodass der pay-off für beide Akteure lediglich zwei (jeweils 6-4=2) betragen würde. In diesem Spiel liegen also zwei Nash-Equilibria vor (Bereitstellung/keine Bereitstellung=6/2 und keine Bereitstellung/Bereitstellung=2/6), die jeweils soziale Optima mit einem Maximalgewinn von acht darstellen. Die Herausforderung der Bereitstellungssituation besteht also lediglich darin, zu koordinieren, dass nicht beide Akteure gleichzeitig die Rolle des best shooters übernehmen (Sandler 2004: 66; Hielscher/Pies 2006). Hat ein potenzieller best shooter hingegen kein ausreichendes Interesse an dem globalen öffentlichen Gut, besteht kein Koordinations-, sondern ein Anreizproblem. So wurde bisher beispielsweise nur unzureichend in die Entwicklung eines Malariaimpfstoffes investiert, weil Malaria keine Bedrohung für die westlichen Industrieländer darstellt und deren Heilung nur geringe Gewinne für die Pharmaindustrie verspricht (vgl. Sandler 2002: 100).

Zusammenfassend kann festgehalten werden, dass die unterschiedlichen Aggregationstechnologien in der hier ausgewählten Literatur mit ganz unterschiedlichen Bereitstellungsprognosen in Verbindung gebracht werden. Eine optimale Versorgung mit weakest-link- und best-shot-Gütern ist in der Regel wahrscheinlicher als mit summation-Gütern. Allerdings sind hierbei weitere Erklärungsvariablen zu berücksichtigen.

20 Dies gilt nur für diskrete best-shot-Güter, zu denen die oben genannten Beispiele zählen. Diskrete Güter sind nicht in diskreten Einheiten verfügbar (eine halbe Brücke ist keine Brücke). Bei stetigen best-shot-Gütern, die sich aus mehreren Einheiten zusammensetzen, besteht hingegen wie bei summation-Gütern die Gefahr der Unterbereitstellung, weil der best shooter nicht die spillover benefits seiner weiteren Mengeneinheit für die anderen Akteure in Rechnung stellen kann. Eine Lösung liegt im Ausschluss der anderen Akteure, zum Beispiel mittels der Patentierung von Arzneimitteln (vgl. Sandler 2003: 136). 


\subsection{Gruppenmerkmale}

Neben den nachfrage- und produktionsseitigen Eigenschaften der Güter selbst, gibt eine Reihe von Eigenschaften der Akteursgruppen Aufschluss über die Wahrscheinlichkeit inter- und transnationaler Kooperation bei der Bereitstellung globaler öffentlicher Güter. Einige dieser Gruppenmerkmale bedürfen hier keiner ausführlicheren Darlegung. So ist durch die rational-choice-Forschung im Allgemeinen und mit Bezug auf die IB insbesondere durch die spieltheoretisch fundierte Regimetheorie (vgl. Keohane 1984; Oye 1986; Zürn 1992; Zangl 1999) bekannt, dass beispielsweise eine langfristige Interaktionsdauer der Akteure nicht nur Reziprozität und den »Schatten der Zukunft« (Axelrod 1987) als Voraussetzungen für die Durchsetzung kooperativer Lösungen begünstigt, sondern auch die Entwicklung von Normen und sozialen Konventionen ermöglicht, die eine Transaktionskosten minimierende Funktion haben (Hardin 1982; North 1990; Ostrom 1990). Auch die mit einer dauerhaften Interaktion einhergehenden Faktoren Nicht-Anonymität der Akteure und Gruppenidentifikation haben einen positiven Effekt auf kooperative outputs im Sinne der Güterbereitstellung (Frey/Bohnet 1996).

Während diese Einflussgrößen von den Autorinnen und Autoren der hier betrachteten Studien ohnehin nur en passant behandelt und nicht explizit in die Analysen integriert werden, finden die Variablen Gruppengröße und Heterogenität (von Einkommen, Präferenzen, Nutzenverteilungen und Bereitstellungskosten) eine größere Beachtung. Beide Variablen sind in Bezug auf globale öffentliche Güter besonders relevant, weil deren Bereitstellung oft durch große Gruppen mit einer Vielzahl von heterogenen governance-Akteuren gewährleistet werden muss. Die Autorinnen und Autoren folgen grundsätzlich der weithin bekannten Annahme Olsons, dass die Suboptimalität der Bereitstellung eines öffentlichen Guts mit steigender Gruppengröße zunimmt (Olson 1965: 22-52), qualifizieren diese Annahme jedoch in mehrfacher Hinsicht. ${ }^{21}$ Während Holzinger auf die methodische Schwierigkeit verweist, den Ef-

21 Olson (1965: 22-52) zufolge können sich große (bzw. »latente«) Gruppen nicht mit einem öffentlichen Gut versorgen, weil es in ihnen aus Sicht des Einzelnen rational erscheint, die Position des Trittbrettfahrers einzunehmen, da sein Beitrag erstens keine spürbare Wirkung erzielt und er umsonst in den Konsumgenuss des öffentlichen Guts kommt und zweitens sein Trittbrettfahren in einer großen Gruppe weniger auffällt und daher nicht sanktioniert wird. Auch in kleinen (»privilegierten «) Gruppen besteht für Olson eine Tendenz zur suboptimalen Versorgung mit dem Kollektivgut. Allerdings sind solche Gruppen durch stärkere Interdependenzbeziehungen gekennzeichnet, so dass ein Nicht-Beitrag des Einzelnen eher mit Reputationsverlusten einhergeht. In kleinen Gruppen produziert der Einzelne zudem einen relativ betrachtet größeren Anteil des Gesamtnutzens als in großen Gruppen, was wiederum bedeutet, dass (unter der Annahme einer Gleichverteilung der Nutzen) sein Grenznutzen höher ist als in großen Gruppen und er somit weniger wahrscheinlich eine private Konsummöglichkeit finden wird, die einen noch höheren Grenznutzen erzeugt als sein Beitrag zum Kollektivgut. Zum anderen - und dieser Gedanke wurde von der Theorie hegemonialer Stabilität aufgegriffen - kann der Nutzen eines Einzelnen aus dem Kollektivgut aber auch so groß sein, dass er die Kosten der Kollektivgutproduktion übersteigt und der Einzelne das Gut in der Folge unilateral bereitstellt, die Gruppe also deshalb »privilegiert« ist (vgl. Olson 1965: 22-52; Hardin 1982: 38-49). Wie oben erwähnt, ging Olson von der Annahme einer summation-Aggregationstechnologie aus, so dass seine Prognosen 
fekt der Variable der Gruppengröße überhaupt isolieren zu können, weil dieser davon abhängt, wie andere Faktoren (z.B. Kosten- und Allokationsfunktionen) durch eine Veränderung der Akteursanzahl beeinflusst werden (Holzinger 2008: 40), verdeutlicht Sandler, dass die Annahme Olsons für viele Bereitstellungssituationen und Arten globaler öffentlicher Güter revidiert werden muss. So hat eine zunehmende Gruppengröße beispielsweise keinen negativen Einfluss auf die Bereitstellung von bestshot-Gütern oder Netzwerkgütern (z.B. Kommunikationssysteme), bei denen zusätzliche Nutzer den Nutzen des Einzelnen erhöhen. Zudem hängt die Validität der Gruppengrößenannahme von individuellen Nutzen-Kosten-Differenzen, Interaktionsanzahlen und Akteurspräferenzen ab (Sandler 2004: 33-34) - Variablen, die bei Olson nicht weiter konkretisiert werden.

Dort jedoch, wo sowohl Barrett als auch Sandler der Gruppengröße neben anderen Variablen einen Einfluss auf die Güterbereitstellung zusprechen (etwa zur Erklärung des Scheiterns beim globalen Klimaschutz) (vgl. Barrett 2007: 74-102; Sandler 2004: 224), beziehen sie sich auf die nominale Gruppengröße. Das muss verwundern, bleiben sie damit doch hinter der Erkenntnis der Regimetheorie zurück, dass nicht die nominale, sondern die $k$-Gruppengröße entscheidend für die Wahrscheinlichkeit der Güterbereitstellung ist (vgl. Snidal 1985b: 598-612). In seiner Kritik an Olsons Gruppenlogik hatte bereits Hardin darauf hingewiesen, dass die Prognose für die Bereitstellung eines öffentlichen Guts weniger von der Gesamtgröße der Gruppe abhänge, als von der Mindestzahl an Gruppenmitgliedern, die von der Kooperation untereinander selbst dann noch profitieren, wenn alle anderen Gruppenmitglieder als Trittbrettfahrer auftreten (Harding 1982: 41-42). Wenn diese, nach Schelling als $k$-Gruppengröße bezeichnete, Mindestzahl relativ klein ist (Schelling 1973), könnten theoretisch sogar nominal große Gruppen mit einem reinen öffentlichen Gut versorgt werden. ${ }^{22} \mathrm{Je}$ kleiner diese $k$-Gruppe ist, desto weniger werden ihre Mitglieder aufgrund der damit verbundenen Interdependenzdichte durch Trittbrettfahren aus der Kooperation ausscheren. Spieltheoretisch betrachtet liegt dann innerhalb dieser Subgruppe keine Dilemmastruktur, sondern ein Koordinationsspiel vor. Die $k$-Gruppe wird umso kleiner, je mehr Heterogenität hinsichtlich der Nutzenerwartungen innerhalb der Gesamtgruppe vorliegt, denn es werden sich nur jene Akteure zur $k$-Gruppe formieren, die einen ungleich höheren Nutzen aus dem öffentlichen Gut ziehen als die übrigen Gruppenmitglieder (ansonsten würden sie deren Trittbrettfahren nicht in Kauf nehmen) ${ }^{23}$ Bei Sandler bleibt das $k$-Gruppenargument jedoch unberücksichtigt, da eine solche Bereitstellungssituation immer durch Disproportionalität in der Lastenteilung und damit durch Pareto-Ineffizienz gekennzeichnet ist (Sandler 2004: 204),

zum Einfluss der Gruppengröße ausschließlich in Bezug auf summation-Güter sinnvoll sind.

22 Sowohl Schelling (1973) als auch Hardin (1982: 41-42) gingen bei ihren Ausführungen zum $k$-Gruppenargument von summation-Gütern aus.

23 Insofern, als dass k $>1$ sein kann und trotzdem kollektives Handeln im Sinne der Bereitstellung öffentlicher Güter möglich sei, ist das - vor allem in der Regimetheorie explizierte - $k$-Gruppen-Argument auch eine Kritik an der (sich auf Olson stützenden) Theorie hegemonialer Stabilität, derzufolge nur ein einzelner Akteur $(k=1)$ das Problem kollektiven Handelns überwinden könne (vgl. Snidal 1985b: 598-612). 
sodass die Versorgung mit einem globalen öffentlichen Gut - zumindest langfristig - nicht durch eine $k$-Gruppe gewährleistet werden kann.

Neben der Gruppengröße ist es vor allem das Gruppenmerkmal Heterogenität, dem die Autorinnen und Autoren der hier untersuchten Studien eine zentrale Bedeutung beimessen. Dabei kann sich Heterogenität auf ganz unterschiedliche Sachverhalte beziehen. Während gemeinhin angenommen wird, dass Heterogenität die Güterbereitstellung begünstigt (Martin 1995: 71-91) - etwa dann, wenn ein Akteur den Nutzen eines globalen öffentlichen Gutes für sich höher bewertet als andere Akteure und die Güterbereitstellung deshalb bei entsprechender Ressourcenausstattung übernimmt zeigen die hier ausgewählten Studien, dass diese Annahme je nach Sachverhalt differenziert zu betrachten ist. Holzinger nutzt beispielsweise die Heterogenität vs. Homogenität von Akteurspräferenzen für globale öffentliche Güter als entscheidendes Kriterium für die Beantwortung der Frage, ob sich die strategische Konstellation ändert, wenn diese Güter nicht nur in einem Einebenensystem (z.B. nur nationale oder nur globale Ebene), sondern im Rahmen eines Mehrebenensystems (wie z.B. der EU) bereitgestellt werden müssen, das sich aus Teilkollektiven mit jeweils eigener governance-Struktur zusammensetzt: Sind die Akteurspräferenzen auf allen Ebenen in gleicher Weise homogen oder heterogen, so ändert sich nichts. Zu Änderungen der strategischen Konstellation kann es aber dann kommen, wenn sich die Akteurspräferenzen zwischen den verschiedenen Ebenen unterscheiden (Holzinger 2008: 174-178; Putnam 1988; Zangl 1999; 1994). Damit ist Holzingers Arbeit die einzige der hier untersuchten Studien, die einen expliziten Mehrebenenvergleich vornimmt und so zumindest im Ansatz der Erkenntnis gerecht zu werden versucht, dass die Bereitstellung globaler öffentlicher Güter in der Regel auf einem komplexen grenz-, sektor- und gruppenüberschreitenden Produktionsprozess beruht (vgl. Kaul/Conceição 2006: 12-15; Kaul/Kocks 2003: 45-49). So zeigt Holzinger beispielsweise anhand der Kapitalbesteuerung in der EU, dass sich die Präferenzen der EU-Bevölkerung deutlich von denen ihrer Regierungen für dieses Gut unterscheiden. Einem homogenen Gesamtkollektiv (alle EU-Bürgerinnen und -Bürger lehnen eine Kapitalbesteuerung ab) steht eine heterogene Gruppe von Repräsentanten gegenüber (einige Regierungen bevorzugen eine Steuerkoordinierung, andere hingegen den Steuerwettbewerb). Für die Bevölkerung ergibt sich ein Gefangenendilemma, in dem Steuerflucht die dominante Strategie ist, die aber zu einer Unterversorgung mit gewünschten öffentlichen Gütern führen kann (Holzinger 2008: 190-194). Für die Regierungen untereinander ergibt sich hingegen ein asymmetrisches Gefangendilemma, das neben dem Defektionsproblem auch ein Verteilungsproblem beinhaltet: Die dominante Strategie der Steuerkoordinierungsgegner ist die Vermeidung einer EU-weit harmonisierten Kapitalertragsteuer. Die Steuerkoordinierungsbefürworter präferieren hingegen eine gleichmäßige Besteuerung, aber nur wenn die Gegner sie auch erheben. Unterlassen es die Gegner, ist die Besteuerung auch keine Option für die Befürworter. Würden sich beide auf die Option der gleichmäßigen Besteuerung einigen, hätten nur die Gegner einen Anreiz, im Nachhinein noch zu defektieren. Darüber hinaus liegt ein Verteilungsproblem vor, weil die pareto-optimale Steuerkoordination zwar die erste Präferenz für die Befürworter, aber nur die zweite Präferenz für die Gegner 
darstellt. Die Gegner präferieren in jedem Fall die Nichtharmonisierung der Besteuerung - selbst dann, wenn die Befürworter sie vornehmen würden. Insofern unterscheidet sich die Spielsituation in zweifacher Hinsicht von einem symmetrischen Gefangenendilemma, in dem alle Regierungen einen Anreiz zum defektieren hätten, jedoch kein Verteilungsproblem vorliegt (Holzinger 2008: 98-99). ${ }^{24}$

Die Heterogenität der Akteure im Mehrebenensystem verändert demnach die strategische Konstellation gegenüber der Einebenenstruktur. Was aber bedeutet das für die Bereitstellung der Steuerharmonisierung als öffentliches Gut? Übt die Heterogenität der Akteurspräferenzen einen positiven oder negativen Effekt auf die Güterproduktion aus? Hier scheint die Antwort weniger eindeutig auszufallen: Für einen positiven Effekt spricht, dass sich die Steuerkoordinierungsbefürworter unter den Repräsentanten mit der 2005 in Kraft getretenen europäischen Richtlinie zur Zinsbesteuerung gegenüber der EU-Bevölkerung durchsetzten. ${ }^{25}$ Obwohl Letztere das Gut ablehnten, wurde es den Präferenzen einiger Repräsentantinnen und Repräsentanten entsprechend bereitgestellt. Für einen negativen Effekt spricht hingegen, dass es 35 Jahre gedauert hat, bis sich die Repräsentanten untereinander auf dieses Abkommen einigen konnten. Auch stellt die Richtlinie nur einen Kompromiss dar, der die Heterogenität der Akteurspräferenzen zwischen jenen Regierungen widerspiegelt, die den Nutzen aus Kapitalzuflüssen höher gewichten (Koordinierungsgegner) und jenen, die die Vermeidung von Steuerflucht und Kapitalabflüssen (Koordinierungsbefürworter) präferieren (Holzinger 2008: 85-99; Genschel 2002). Dieser nicht eindeutige Effekt legt die Vermutung nahe, dass Heterogenität (ähnlich wie die Gruppengröße) nur einen bedingten Effekt ausübt. So ist beispielsweise anzunehmen, dass die Repräsentantinnen und Repräsentanten ihre heterogenen Präferenzen nur unter der Bedingung ungleicher Machtverteilungen und spezifischer institutioneller Merkmale (wie etwa der Stimmgewichtung und der Einstimmigkeitsregel im Ministerrat) an der Bevölkerung vorbei durchsetzen konnten.

Während wie Holzinger die meisten der hier untersuchten Beiträge vor allem die Heterogenität von Präferenzen (aber auch von Einkommen und Bereitstellungskosten) in ihre Analysen integrieren, rücken Kaul und Mendoza einen weiteren zentralen Aspekt in den Vordergrund: die Heterogenität in der Verteilung der Nutzen eines öffentlichen Guts. So ist es nicht selten der Fall, dass Akteure ein öffentliches Gut zwar in gleicher Weise präferieren und auch prinzipiell Zugang zu diesem Gut haben (kein Ausschluss), aber die Verteilung der aus dem Gut erwachsenden Vorteile heterogen ist. Ein Beispiel auf nationaler Ebene ist das Rechtssystem, das Frauen lange Zeit nicht dieselben Rechte eingeräumt hat wie Männern (Kaul/Mendo-

24 Im symmetrischen Gefangenendilemma würden die Regierungen defektieren, sobald ein Abkommen erreicht ist, zugleich aber eine gemeinsame Steuer gegenüber dem NashEquilibrium keine Besteuerung bevorzugen. Im vorliegenden asymmetrischen Gefangenendilemma ist es hingegen einfacher, compliance nach einem Abkommen zu gewährleisten, weil nur ein Staat einen Anreiz zum Defektieren hat, aber es ist schwieriger, überhaupt erst ein Abkommen zu erzielen (Holzinger 2008: 99).

25 Die Richtlinie zielt auf eine ausnahmslose und gleichmäßige Besteuerung der Zinseinnahmen aller EU-Bürger mit EU-Wohnsitz (unabhängig davon, wo das Zinseinkommen erwirtschaftet wird) ab. 
za 2003: 91-93). Überträgt man diesen Gedanken auf die oben dargestellte Peacekeeping-Fallstudie von Sandler (2004), ließe sich mittels einer konkurrierenden und ebenfalls empirisch zu prüfenden Hypothese argumentieren, dass nicht nur private Nutzen einen Anreiz für die Bereitstellung von Friedenseinsätzen darstellen, sondern auch heterogen verteilte öffentliche Nutzen. So ist denkbar, dass eine Staatengruppe aufgrund ihrer Konfliktnähe mehr von der Verhinderung von Flüchtlingsströmen (reines öffentliches Gut) profitiert und deshalb eine Friedensoperation ungleich stärker unterstützt als der Rest der bereitstellenden Akteure. Auch hier ist der Einfluss der Heterogenität wiederum bedingt durch weitere Variablen wie etwa der Ressourcenausstattung. Wird das genaue Zusammenspiel solcher Variablen nicht berücksichtigt, könnte man zum selben Ergebnis wie Snidal kommen: »The impact of heterogeneity is heterogeneous ( Snidal 1995: 63, zitiert in Holzinger 2008: 84).

\subsection{Merkmale der rechtlichen, institutionellen und sozialen Umwelt}

Kooperation im Sinne der Güterbereitstellung kann den Studien zufolge weder durch die ausschließliche Betrachtung der Gütereigenschaften noch durch die ausschließliche Betrachtung der Gruppenmerkmale, sondern nur durch deren Zusammenspiel mit den spezifischen rechtlichen, institutionellen und sozialen Kontexten der Bereitstellungssituation erklärt werden. Jede Veränderung dieser Kontextfaktoren kann wiederum zu Veränderungen in der Kosten-Nutzen-Kalkulation der Akteure, ihrer strategischen Interaktion und damit der Güterbereitstellung führen. Die experimentelle Forschung zu öffentlichen Gütern, aber auch institutionalistisch fundierte (IB-)Beiträge haben in der Vergangenheit wichtige Erkenntnisse über den Effekt von Regeln und Institutionen auf die Anreizstruktur bei der Bereitstellung öffentlicher Güter geliefert, die dann wiederum als Vorschläge für die Lösung der Güterproblematik auf globaler Ebene genutzt werden können (siehe Abschnitt 3): Dauerhaft institutionalisierten Kommunikations- und Sanktionsmöglichkeiten wird dabei beispielsweise ebenso ein positiver Effekt zugesprochen (Ledyard 1995) wie operativen Regeln, die die Entscheidungen der Akteure in einer Handlungssituation strukturieren (Ostrom et al. 1994). ${ }^{26}$ Die Zuteilung von Eigentumsrechten kann Beiträge zu globalen öffentlichen Gütern befördern (Sandler 2004: 259), und Regime reduzieren die Transaktionskosten bei der Güterbereitstellung (Keohane 1984).

Über diese Erkenntnisse hinaus tragen die hier diskutierten Beiträge mit einer Vielzahl von Fallstudien zu einer weiteren Spezifizierung unterschiedlicher potenziell relevanter Kontextfaktoren bei. Beispielsweise zeigt Holzinger (2008: 112-122), dass der Umfang der Bereitstellung von credit ratings (ratings über die Kreditwürdigkeit von Staaten und Unternehmen) als globales öffentliches Gut mit unterschiedlichen

$26 \mathrm{Zu}$ solchen operativen Regeln gehören Aggregationsregeln, die bestimmte Entscheidungsverfahren festlegen und Informationsregeln, die spezifizieren, welche Informationen den Akteuren zukommen müssen (vgl. Ostrom et al. 1994: 41-42). 
ökonomischen und politischen Rahmenbedingungen variiert: ${ }^{27}$ In einer frühen Phase des Kapitalmarktes, der durch ausreichendes Kapitalangebot und die Abwesenheit von rating-Agenturen gekennzeichnet war, hatten die Kreditnehmer selber keinen Anreiz, ratings einzuführen. Mit zunehmend knapper werdendem Kapitalangebot und dem Aufkommen von rating-Agenturen (initiiert durch Investoren) stellen die Kreditnehmer jedoch zunehmend selber die ratings über Agenturen bereit, weil ihnen ohne rating aufgrund von Investorenentscheidungen oder staatlicher Regulierung nur ein begrenzter Kapitalzugang möglich ist. Die erfolgreiche Bereitstellung des Guts konnte also erst vor dem Hintergrund entsprechender rechtlicher und ökonomischer Bedingungen gewährleistet werden. Mit Bezug auf den Umweltschutz als globales öffentliches Gut weist Holzinger zudem nach, dass die Frage, ob der staatliche regulative Wettbewerb um Umweltstandards in ein race to the top oder ein race to the bottom mündet, unter anderem vom vorherrschenden Handelsregime und dem betreffenden Standard abhängt (Holzinger 2008: 122-136). Barretts vergleichende Untersuchung des Montrealprotokolls zur Rettung der Ozon-Schicht und des Kyotoprotokolls zur Ausgestaltung der UN-Klimarahmenkonvention verdeutlicht wiederum den Einfluss unterschiedlicher Enforcement-Mechanismen auf die tatsächliche Güterbereitstellung. Anders als das Kyotoprotokoll beinhaltete das Montrealprotokoll eine Kombination aus sticks and carrots, um die Vertragsdurchsetzung zu gewährleisten: Erstgenannte bestanden darin, den Handel von FCKW und FCKW-haltigen Produkten zwischen Vertrags- und Nichtvertrags-Parteien zu beschränken. Der fehlende Marktzugang für Nichtmitglieder schuf wiederum einen Anreiz, dem Abkommen beizutreten. Carrots kamen darin zum Ausdruck, dass die mit dem Abkommen einhergehenden Mehrkosten der Entwicklungsländer von den Industrieländern übernommen wurden. Diese beiden Faktoren trugen maßgeblich zum erfolgreichen Schutz der Ozonschicht bei (vgl. Barrett 2007: 74-83).

Die Beiträge von Kaul et al. und Albin reichen hingegen über die üblichen rationalistischen Erklärungen hinaus, indem sie einen Zusammenhang zwischen inklusiven, transparent und fair ausgestalteten governance-Arrangements und dem Erfolg der Güterbereitstellung herstellen. Ob sich allerdings die dort implizit formulierte Hypothese bestätigt, dass ein globales öffentliches Gut umso wahrscheinlicher bereitgestellt wird, je mehr die potenziell von diesem Gut betroffenen Akteure am Entscheidungsprozess beteiligt werden (Inklusivität) und je demokratischer und partizipativer dieser ist, kann nur durch weitere empirische Untersuchungen geklärt werden (vgl. Kaul et al. 2003b; Albin 2003).

27 Rating-Analysen sind nicht-rivalisierend, weil ihre individuelle Nutzung nicht die Verfügbarkeit für andere Investoren mindert. Zugleich sind sie entweder bewusst nicht-ausschließbar auf öffentlichen web sites verfügbar oder ein Konsumausschluss ist kaum möglich, weil sie in Fachjournalen veröffentlicht werden, die faktisch auch für Nicht-Zahler weltweit zugänglich sind. 


\section{Generalisierbare Empfehlungen zur Lösung der Kollektivhandlungsprobleme}

Abschließend soll kurz in den Blick genommen werden, welche generalisierbaren Empfehlungen sich aus den Forschungsergebnissen für die Lösung der Kollektivhandlungsprobleme und damit für die Optimierung der Bereitstellung globaler öffentlicher Güter ergeben. Die Autorinnen und Autoren der hier besprochenen Studien warten mit einer Vielzahl von Lösungsvorschlägen auf. Sie sind sich dabei aber bewusst, dass es angesichts der Fülle variierender Bereitstellungsmerkmale, die das Handeln der Akteure beeinflussen, nicht die eine Lösung für das globale öffentliche Güterproblem gibt: »The view, that there is a common fix, institutional innovation, or blueprint treaty is doomed to fail« (Sandler 2004: 43-44). Dennoch sind generalisierbare Empfehlungen für solche Fälle möglich, die eine identische Kombination an Bereitstellungsmerkmalen und damit einhergehend eine identische strategische Konstellation aufweisen. In der Studie von Holzinger resultieren beispielsweise aus 30 Kombinationen von Bereitstellungsmerkmalen über alle Fallstudien hinweg sieben unterschiedliche strategische Interaktionen (Spiele). Diese legen wiederum drei unterschiedliche Arten von Kollektivhandlungsproblemen offen: Effizienz-, Koordinations- und Verteilungsprobleme. Die Suche nach Lösungen richtet sich dann nicht auf die verschiedenen Bereitstellungsmerkmale, sondern auf die resultierenden Kollektivhandlungsprobleme. Koordinationsprobleme (z.B. Versicherungsspiel) und Einigungsprobleme (z.B. chicken-game) sind in der Regel einfacher zu lösen als Dilemmata (z.B. Gefangenendilemma) oder Verteilungsprobleme (z.B. Nullsummenspiel) (vgl. Holzinger 2008: 137-159). Diese Verschiedenheit an Kollektivhandlungsproblemen macht deutlich, dass sich die Bereitstellung (globaler) öffentlicher Güter keineswegs ausschließlich als Dilemma darstellt (vgl. Mueller 2003: 9-17; Hardin 1982: 28-30). Ist erst einmal bekannt, welche Situationen des grenzüberschreitenden Regierens mit welchen spezifischen Handlungsproblemen verbunden sind und dies aufzuzeigen ist der originäre Beitrag der Theorie -, steht eine Reihe von Lösungsvorschlägen angrenzender Disziplinen (Institutionenökonomik, Regimetheorie, compliance-Forschung) zur Verfügung. Während die verschiedenen oben genannten Kollektivhandlungsprobleme und deren Lösungen insbesondere im Rahmen des situationsstrukurellen Ansatzes der Regimetheorie bereits umfangreich erforscht wurden (vgl. Martin 1992; Zürn 1992; Zangl 1999), liegt der Zugewinn der Theorie der globalen öffentlichen Güter darin, aufzuzeigen, auf welche genauen Merkmale der Bereitstellungssituation diese Handlungsprobleme zurückzuführen sind.

Koordinationsprobleme lassen sich in der Regel bereits durch Kommunikation zwischen den Akteuren lösen. In Verhandlungen können die Akteure ihr Handeln so koordinieren, dass kein individueller Anreiz mehr besteht, von einer pareto-optimalen Lösung abzuweichen. Wenn Koordination allerdings durch heterogene Kosten-Nutzen-Verteilungen erschwert wird - etwa dann, wenn einzelne Akteure höhere oder zu hohe Bereitstellungskosten haben oder einen niedrigeren Nutzen aus dem Gut ziehen, sind zusätzliche redistributive oder kompensatorische Maßnahmen vonnöten, um die Versorgung mit dem globalen öffentlichen Gut sicherzustellen (Holzinger 2001: 133): 
Hängt die Bereitstellung eines weakest-link-Guts wie der Seuchenbekämpfung von einem Entwicklungsland ab, kann unter Umständen ein Ressourcentransfer hilfreich sein. ${ }^{28}$ Verfügt ein potentieller best shooter zwar über die Technologie, ein bestshot-Gut bereitzustellen, präferiert es aber nicht, oder kann er seine Forschungs- und Entwicklungskosten nicht alleine schultern, liegt die Lösung in Anreiz- oder Kompensationszahlungen durch die anderen Akteure (vgl. Kaul/Conceição 2006: 15).

Für die Lösung von Dilemmata und Verteilungsproblemen sind Kommunikation und Informationen nicht ausreichend. Hierfür bedarf es weiterer institutioneller Mechanismen, um das Defektieren der Akteure zu verhindern und Einigung, Überwachung und Sanktion zu gewährleisten. Die Lösungsvorschläge angrenzender Disziplinen aufgreifend, bietet die Studie von Holzinger (2008) einen guten Überblick, wie governance-Strukturen ausgestaltet sein sollten, um Dilemma-, Verteilungs- und Einigungsprobleme beheben zu können: Feste Entscheidungsregeln und die Nutzung des Mehrheitsprinzips ohne Vetorecht werden als ebenso vielversprechend für die Lösung von Einigungsproblemen betrachtet, wie die Übertragung von legitimer Sanktionsmacht und von Überwachungskapazitäten an Institutionen des globalen Regierens für die Lösung von Dilemmata (Holzinger 2001: 9-10; Zürn 1992; Zangl 1999). Darüber hinaus bieten die Beiträge von Sandler und Barrett eine Vielzahl ganz konkreter und aus den Fallstudien gewonnener Vorschläge für die Lösung der jeweiligen Kollektivhandlungsprobleme. So heben beispielsweise beide Autoren den positiven Effekt von Beitrags- und Teilnehmerschwellen (thresholds) auf das kollektive Handeln hervor: Das Setzen einer Schwelle, die erreicht werden muss, damit das Gut bereitgestellt werden kann, schafft einen Anreiz zur Überwindung des Defektierens. Internationale Abkommen, die erst in Kraft treten, wenn sie von einer bestimmten Anzahl von Staaten ratifiziert sind (Sandler 2004: 93), basieren ebenso auf diesem Mechanismus wie verschiedene Finanzierungsfonds für globale öffentliche Güter, die erst ausgeschüttet werden, wenn eine bestimmte Beitragssumme erreicht ist. Als zentral für die Überwindung des Trittbrettfahrerproblems erachten die Autoren und Autorinnen zudem die Schaffung selektiver Anreize, die - wie im Falle von joint product-Gütern - individuelle Gewinne in Aussicht stellen und damit Beiträge zum öffentlichen Gut begünstigen (vgl. Buttkereit 2009). So hält Barrett ein Abkommen zur Reduzierung von Rußemissionen für leichter realisierbar als ein Abkommen zur Reduzierung von $\mathrm{CO}_{2}$-Emissionen, weil es nicht nur zur Verbesserung des globalen Klimaschutzes, sondern auch zur Verbesserung der lokalen Luftqualität beiträgt (Barrett 2004: 49). Neben weiteren Faktoren messen die Autorinnen und Autoren zudem klar definierten Eigentumsrechten eine Bedeutung für den Schutz von Allmendegütern zu (Sandler 2004: 259). Beispielsweise könnte die Ausweitung nationaler Fischereizonen in den Meeren eine bessere Regulierung der Fangquoten ermöglichen.

28 Dies gilt dann, wenn das dem weakest-link-Gut zugrunde liegende Problem ein Ressourcenproblem darstellt. Handelt es sich hingegen um ein governance-Problem, wäre eine institutionelle Hilfestellung - ein Bindungsservice - erforderlich. 


\section{Forschungsdefizite und zukünftige Entwicklungslinien}

Die in diesem Literaturbericht betrachteten Studien verdeutlichen, dass die Theorie der (globalen) öffentlichen Güter eine erstaunliche Entwicklung durchlaufen hat. Ursprünglich als Teil der normativen neoklassischen Wohlfahrtsökonomie entwickelt, die aufzuzeigen versuchte, was kollektiv rationales und effizientes Verhalten wäre, wird sie hier als positive Theorie zur Erklärung realen Akteursverhaltens bei der Bereitstellung globaler öffentlicher Güter genutzt. Die Stärke der Theorie ist dabei ohne Zweifel, die Grundstruktur globaler Handlungsprobleme offenzulegen: Ohne den Begriff der globalen öffentlichen Güter, der einen gemeinsamen Nenner für ansonsten ganz unterschiedliche Phänomene bietet, würde ein Vergleich - zum Beispiel zwischen Klimaschutz und Terrorismusabwehr - kaum möglich sein. Diese Abstraktionsleistung ermöglicht es, empirisch beobachtbare globale Politikphänomene theoriegeleitet zu systematisieren und zu prognostizieren, wann und in welchem Umfang Akteure des globalen Regierens bereit sind, einen Beitrag zur Bereitstellung globaler öffentlicher Güter zu leisten.

Dabei zeigen die hier untersuchten Studien, dass es keineswegs zwangsläufig zum kollektiven Versagen von governance-Akteuren bei der Versorgung mit globalen öffentlichen Gütern kommen muss. Vielmehr machen die Autorinnen und Autoren eine Vielzahl unterschiedlicher Güter- und Gruppenmerkmale sowie Merkmale der rechtlichen und institutionellen Umwelt für die Kooperationswahrscheinlichkeit bei der Bereitstellung dieser Güter verantwortlich - und je nachdem welche Merkmale vorliegen, sind durchaus positive Bereitstellungsszenarien möglich. Die weltweite Realisierung von Flughafenkontrollen zur Terrorismusabwehr lässt sich mit dem weakestlink-Charakter dieses Guts erklären und Erfolge in der Gesundheitsvorsorge mit global verfügbaren best-shot-Ergebnissen der medizinischen Grundlagenforschung. Umfangreich unterstützte Friedensoperation lassen sich auf den privaten Anteil ihrer joint-product-outputs oder heterogen verteilte öffentliche Nutzen zurückführen, und der Erfolg beim Schutz der Ozonschicht basierte unter anderem auf der spezifischen Ausgestaltung des Montrealprotokolls als rechtlichem Merkmal der Bereitstellungssituation.

Alle hier betrachteten Studien zielen darauf ab, ihre theoriegeleiteten Prognosen mit der Empirie zu konfrontieren - wenngleich oftmals noch mehr veranschaulichend als wirklich testend. Sollten sich die verschiedenen Erklärungsvariablen auch über den derzeitigen Forschungsstand hinaus als empirisch plausibel behaupten, hat die Theorie der globalen öffentlichen Güter durchaus das Potenzial, den empirischen Gehalt des rationalistischen IB-Forschungsprogramms zu erweitern. ${ }^{29}$ Kritikwürdig

29 Nach Lakatos (1982) würde die Theorie somit zu einer progressiven Problemverschiebung des rationalistischen IB-Forschungsprogramms beitragen. Folgt man der Argumentation von Johannes Marx, dass sich die handlungstheoretisch fundierten Großtheorien der Internationalen Beziehungen entweder dem soziologischen oder dem ökonomischen Forschungsprogramm zuordnen lassen, dann ist ihr ontologisches Unterscheidungskriterium dabei ausschließlich ihr Handlungsgesetz (Logik der Konsequenzialität vs. Logik der Angemessenheit), das zugleich den harten Kern des jeweiligen Forschungsprogramms bildet 
ist allerdings, dass die Studien trotz ihrer prinzipiell empirischen Ausrichtung zugleich noch stark vom analytischen Zweig des ökonomischen Forschungsprogramms mitsamt seiner modellanalytischen Orientierung geprägt sind und dabei von Grundannahmen ausgehen, die einer kritischen Überprüfung nicht standhalten können (Hyperrationalität, vollständige Informationen, etc.). So verwundert es zum Beispiel auch nicht, dass keine der hier untersuchten Studien eine direkte Messung von Nutzenfunktionen vornimmt. Stattdessen wird, wie auch in anderen rationalistischen IBTheorien, implizit von fixen (insbesondere Wohlfahrts-) Interessen ausgegangen, die zumeist in Form von Post-hoc-Erklärungen aus dem Akteurshandeln abgeleitet werden. Eine solche Vorgehensweise ist nicht nur mit dem üblichen revealed preference problem behaftet (vgl. Deitelhoff 2006: 87; Mayer 2006: 118). Sie ist insbesondere deshalb abzulehnen, weil die Setzung fixer Präferenzen eine axiomatische Zusatzannahme darstellt, nach Lakatos aber alle Zusatzannahmen empirisch zu erheben sind (Lakatos 1982: 47-50). So gibt sich auch die Theorie der globalen öffentlichen Güter hier mit analytischen Setzungen zufrieden, wo empirische Untersuchungen notwendig wären. Kennzeichnend für einige der untersuchten Studien ist zudem, dass sie den Rationalitätsbegriff auf das Prinzip des Eigennutzes einengen und damit Handlungsmotive wie Altruismus (vgl. Marx 2006: 106) ausschließen. Eine solche analytisch gesetzte Einengung auf egoistisch determinierte Nutzenkalküle findet sich bereits bei Olson (1965) und gilt hier vor allem für die Beiträge von Sandler $(2003 ; 2004){ }^{30}$ Ebenfalls aus forschungspragmatischen Gründen wird zudem häufig auf ein objektives Rationalitätsverständnis zurückgegriffen - insbesondere dann, wenn die prognostizierten strategischen Akteurskonstellationen spieltheoretisch modelliert werden. So baut auch die spieltheoretisch fundierte Darlegung der Studie von Holzinger auf der analytischen Randbedingung vollständiger Informationen der Akteure auf, auch wenn »often this condition will not be fulfilled for real actors« (Holzinger 2008: 29).

In der Gesamtschau der hier besprochenen Literatur wird zudem deutlich, dass sich die meisten der Studien durch eine starke Anwendung der Ceteris-paribus-Klausel auszeichnen. Zwar ist es sicherlich als Leistung und als wichtiger Beitrag zur Fortentwicklung der positiven Theorie der globalen öffentlichen Güter zu betrachten, dass die Autorinnen und Autoren zunächst die verschiedenen Einflussgrößen (vornehmlich) isoliert betrachten, um so ihren jeweiligen Einfluss überhaupt erst ermitteln zu können. Künftige Forschungsarbeiten sollten aber weitere Erkenntnisse über die Be-

(Marx 2006: 18). Zusatzannahmen reichern den harten Kern um empirische Informationen an und bilden die positive Heuristik, deren Zweck es ist, das Forschungsprogramm weiterzuentwickeln und gegen Widerlegungen zu schützen. Solange sie den empirischen Gehalt eines Forschungsprogramms erweitern, ohne dabei im Widerspruch zu seinem harten Kern zu stehen, durchläuft das Forschungsprogramm eine empirisch progressive Problemverschiebung (Marx 2006: 44-51; Humrich 2008: 68-70).

30 Dass diese enge Eigennutzannahme keineswegs repräsentativ für die ökonomische Literatur insgesamt ist, verdeutlichen nicht nur Beiträge der experimentellen Wirtschaftsforschung (siehe z.B. Diekmann 2008; auch Becker 1993), sondern auch ökonomische fundierte Studien zur Bereitstellung öffentlicher Güter (z.B. Smitha et al. 1995; Andreoni 1990). 
reitstellung globaler öffentlicher Güter produzieren können, wenn sie in Form eines holistischen Erklärungsansatzes noch stärker das Zusammenspiel der einzelnen Variablen in den Blick nehmen. Eine solche Vorgehensweise, die in der Studie von Holzinger bereits angelegt ist, würde dabei keineswegs zur völlig überbordeten und nicht mehr erklärungsfähigen »Charybdis hochkomplexer Ansätze« (Wolf 2003: 138) mutieren müssen. So sollte bei einer Weiterentwicklung des Ansatzes der Versuch unternommen werden, noch genauer die Besonderheiten des jeweiligen zu erklärenden empirischen Falls zu betrachten. Nicht alle öffentliche Güter-Bereitstellungsszenarien weisen dieselben Charakteristika auf, sondern es liegt in der Regel eine überschaubare Anzahl verschiedener Merkmale der Bereitstellungssituation vor. Eine stärkere Korrespondenz mit der Empirie könnte es ermöglichen, eine begrenzte Anzahl von Variablen aus der Vielzahl theoretisch möglicher (und damit potenziell infrage kommender) Variablen zu selektieren. Ist ein bestimmtes Set an Erklärungsvariablen erst einmal bekannt, sollte sich deren Zusammenspiel auch im Forschungsdesign berücksichtigen lassen können. Unverzichtbar ist in diesem Zusammenhang auch, noch deutlicher die Unterschiede zwischen der Bereitstellung öffentlicher und globaler öffentlicher Güter herauszuarbeiten. Die Besonderheiten der Mehrebenenpolitik bei der Versorgung mit globalen öffentlichen Gütern und die dabei existierenden unterschiedlichen Handlungslogiken, -restriktionen und -optionen der Akteure auf der supra-, trans- und nationalen Ebene werden anders als in anderen Studien (vgl. Aretz 2006; Zangl 1999) nur unzureichend analysiert. Der eher normativ-programmatisch ausgerichtete Sammelband von Kaul et al. (2003a) bietet wichtig Anhaltspunkte, an welchen Stellen eine solche Analyse genau ansetzen könnte.

Dass noch vielfältige Forschungsmöglichkeiten bezüglich der weiteren empirischen Fundierung der Theorie der globalen öffentlichen Güter gegeben sind, zeigt sich nicht nur dann, wenn das Explanandum die Wahrscheinlichkeit der Kooperation bei der Bereitstellung dieser Güter ist. So nutzt inzwischen eine Reihe von Studien verschiedene der hier vorgestellten unabhängigen Variablen, um auch andere Phänomene des globalen Regierens zu erklären. Beispielsweise spricht Alkuin Kölliker der territorialen und funktionalen Reichweite der durch ein Gut generierten Externalitäten (externe Effekte) einen zentralen Einfluss auf die Art der Formgebung von Institutionen des Weltregierens zu (Kölliker 2006: 206-212). Bei ihm ist das Explanandum somit nicht die Kooperationsbereitschaft der Akteure, sondern die Form der institutionellen Ausgestaltung von Global Governance. ${ }^{31}$ Je nach Reichweite und Zusammenspiel territorialer und funktionaler Externalitäten werden dabei unterschiedliche Formen von governance-Institutionen prognostiziert, die sich hinsichtlich ihrer Inklusivität und Verortung im Mehrebenensystem (national-global) voneinander unterscheiden. Kaul erklärt hingegen die Umstrittenheit globaler öffentlicher Güter (wie z.B. bestimmter WTO-Handelsregeln) mit der Heterogenität in der Verteilung der Nutzen dieser Güter: Je ungleicher die zu erwartende Nutzenverteilung ist, desto umstrittener ist die Frage, ob und zu wessen Kosten ein solches Gut überhaupt in der öffentlichen Domäne platziert sein soll (Kaul 2008: 95-99).

31 Für eine Modifikation des Ansatzes von Kölliker siehe Rittberger et al. (2009: 358-362). 
Bei einer Weiterentwicklung des Ansatzes sollte zudem der Versuch unternommen werden, eine Brücke zwischen rationalistischen und konstruktivistischen Analysen der Bereitstellung globaler öffentlicher Güter zu bauen. Das Gros der global public goods-Forschung - und so auch die hier untersuchten Studien - betrachten Güterbeiträge ausschließlich als Ausdruck strategischen, nicht aber als Ausdruck normbasierten und kommunikativen Handelns. Anstatt empirische Evidenzen beispielsweise für die Rolle normativer Wertverpflichtungen (Aretz 2005: 337-341) und gemeinsamer Identitäten (Bobrow/Boyer 2005: 17-47) bei der Bereitstellung globaler öffentlicher Güter auszublenden, sollte vielmehr kritisch überprüft werden, inwieweit solche Befunde mit den eigenen - gegebenenfalls zu modifizierenden - Annahmen vereinbar sind.

Diese verschiedenen Weiterentwicklungsszenarien könnten dazu beitragen, der Theorie der globalen öffentlichen Güter künftig noch mehr Aufmerksamkeit in den IB zuteil werden zu lassen. In jedem Fall verdient sie ihren Platz in der Disziplin als das, was sie inzwischen ist: eine akteurspluralistische Theorie des Weltregierens, deren theoretischer Mehrwert vor allem darin liegt, aufzuzeigen, unter welchen Bedingungen Akteure einen Beitrag zum grenzüberschreitenden Regieren in Form der Bereitstellung globaler öffentlicher Güter leisten. In diesem Sinne kann die Theorie auch als eine Antwort auf die Schwierigkeiten bestehender IB-Theorien verstanden werden, Global Governance-Prozesse und -Strukturen adäquat zu erklären.

\section{Literatur}

Aggarwal, Vinod K./Dupont, Cedric 1999: Goods, Games, and Institutions, in: International Political Schience Review 20: 4, 393-409.

Albin, Cecilia 2003: Getting to Fairness: Negotiations over Global Public Goods, in: Kaul, Inge/ Conceição, Pedro/Le Goulven, Katell/Mendoza, Ronald U. (Hrsg.): Providing Global Public Goods: Managing Globalization, New York, NY, 263-279.

Andreoni, James 1990: Impure Altruism and Donations to Public Goods: A Theory of Warm Glow Giving, in: The Economic Journal 100: 401, 464-477.

Arce, Daniel G./Sandler, Todd 2002: Regional Public Goods: Typologies, Provision, Financing, and Development Assistance, Stockholm.

Aretz, Hans-Jürgen 2005: Die Relevanz von Wertverpflichtungen bei der Bereitstellung öffentlicher Güter, in: Zeitschrift für Soziologie 34: 5, 326-343.

Aretz, Hans-Jürgen 2006: Globale kollektive Güter und internationale Umweltpolitik. Das Beispiel der Ozonpolitik, Frankfurt a. M.

Axelrod, Robert 1987: Die Evolution der Kooperation, München.

Barrett, Scott 2004: Critical Factors for the Successful Provision of Transnational Public Goods: Background Study for the International Task Force on Global Public Goods, Stockholm.

Barrett, Scott 2007: Why Cooperate? The Incentive to Supply Global Public Goods, New York, NY.

Becker, Gary S. 1993: Nobel Lecture: The Economic Way of Looking at Behavior, in: The Journal of Political Economy 101: 3, 385-409.

Bergstrom, Theodore C./Blume, Lawrence/Varian, Hal 1986: On the Private Provision of Public Goods, in: Journal of Public Economics 29: 1, 25-49.

Bernauer, Thomas 1995: Theorie der Klub-Güter und die Osterweiterung der NATO, in: Zeitschrift für Internationale Beziehungen 2: 1, 79-105. 
Besley, Timothy/Ghatak, Maitreesh 2006: Retailing Public Goods: The Economics of Corporate Social Responsibility, in: Journal of Public Economics 91: 9, 1645-1663.

Bienen, Derk/Freund, Corinna/Rittberger, Volker 1999: Gesellschaftliche Interessen und Außenpolitik. Die Außenpolitiktheorie des utilitaristischen Liberalismus (Tübinger Arbeitspapiere zur internationalen Politik und Friedensforschung), Tübingen.

Bobrow, Davis B./Boyer, Marc A. 2005: Defensive Internationalism: Providing Public Goods in an Uncertain World, Ann Arbor, MI.

Buchanan, James M. 1965: An Economic Theory of Clubs, in: Economica 32: 1, 1-14.

Buttkereit, Sören 2009: Intersectoral Alliances: An Institutional Economics Perspective, Berlin.

Carbone, Maurizio 2007: Supporting or Resisting Global Public Goods? The Policy Dimension of a Contested Concept, in: Global Governance 13: 2, 179-198.

Coleman, James S. 1991: Grundlagen der Sozialtheorie. Handlungen und Handlungssysteme, Band 1, München.

Cornes, Richard/Sandler, Todd 1996: The Theory of Externalities, Public Goods, and Club Goods, New York, NY.

Deitelhoff, Nicole 2006: Überzeugung in der Politik, Grundzüge einer Diskurstheorie internationalen Regierens, Frankfurt a. M.

Diekmann, Andreas 2008: Soziologie und Ökonomie. Der Beitrag experimenteller Wirtschaftsforschung zur Sozialtheorie, in: Kölner Zeitschrift für Soziologie und Sozialpsychologie 60: 3, 528-550.

Efinger, Manfred/Rittberger, Volker/Zürn, Michael 1988: Internationale Regime in den OstWest-Beziehungen. Ein Beitrag zur Erforschung der friedlichen Behandlung internationaler Konflikte, Frankfurt a. M.

Ferroni, Marco/Mody, Ashoka (Hrsg.) 2002: International Public Goods: Incentives, Measurement, and Financing, Norwell, MA.

Frey, Bruno S./Bohnet, Iris 1996: Cooperation, Communication, and Communitarism: An Experimental Approach, in: Journal of Political Philosophy 4: 4, 322-336.

Genschel, Philipp 2002: Steuerharmonisierung und Steuerwettbewerb in der Europäischen Union, Frankfurt a. M.

Gilpin, Robert 1987: The Political Economy of International Relations, Princeton, NJ.

Hardin, Garett 1968: The Tragedy of the Commons, in: Science 162: 12, 1243-1248.

Hardin, Russell 1982: Collective Action, Baltimore, MD.

Hasenclever, Andreas/Mayer, Peter/Rittberger, Volker 1997: Theories of International Regimes, Cambridge, MA.

Hausken, Kjell/Plümper, Thomas 1999: The Impact of Actor Heterogeneity on the Provision of International Public Goods, in: International Interactions 25: 1, 61-94.

Hielscher, Stefan/Pies, Ingo 2006: Internationale Öffentliche Güter. Ein neues Paradigma für die Entwicklungszusammenarbeit?, in: Ebert, Udo (Hrsg.): Wirtschaftsethische Perspektiven VIII. Grundsatzfragen, Unternehmensethik, Institutionen, Probleme internationaler Kooperation und nachhaltiger Entwicklung (Schriftenreihe des Vereins für Sozialpolitik), Berlin, 201-227.

Hirshleifer, Jack 1983: From Weakest Link to Best-Shot: The Voluntary Provision of Public Goods, in: Public Choice 41: 3, 371-386.

Holzinger, Katharina 2001: Aggregation Technology of Common Goods and its Strategic Consequences: Global Warming, Biodiversity, and Siting Conflicts, in: European Journal of Political Research 40: 2, 117-138.

Holzinger, Katharina 2002: Transnational Common Goods: Regulatory Competition for Environmental Standards, in: Héritier, Adrienne (Hrsg.): Common Goods: Reinventing European and International Governance, Lanham, MD, 59-82.

Holzinger, Katharina 2003: Transnationale Gemeinschaftsgüter. Strategische Konstellationen, Kollektivhandlungsprobleme und Mehrebenensteuerung. Max-Planck-Institut zur Erforschung von Gemeinschaftsgütern, Tätigkeitsbericht 2003, Bonn. 
Holzinger, Katharina 2008: Transnational Common Goods: Strategic Constellations, Collective Action Problems, and Multi-Level Provision, Basingstoke.

Humrich, Christoph 2008: Kommunikative Vernunft und Internationale Beziehungen. Eine Theoriearbeit in praktischer Absicht (Universität Bremen: Dissertation), Bremen.

International Task Force on Global Public Goods 2006: Meeting Global Challenges: International Cooperation in the National Interest, Final Report, Stockholm.

Isaac, Mark R./Walker, James/Williams, Arlington W. 1994: Group Size and Voluntary Provision of Public Goods: Experimental Evidence Utilizing Large Groups, in: Journal of Public Economics 54: 1, 1-36.

Kaul, Inge 2008: Providing (Contested) Global Public Goods, in: Rittberger, Volker/Nettesheim, Martin (Hrsg.): Authority in the Global Political Economy, Basingstoke, 89-115.

Kaul, Inge/Conceição, Pedro 2006: Why Revisit Public Finance Today?, in: Kaul, Inge/Conceição, Pedro (Hrsg.): The New Public Finance: Responding to Global Challenges, New York, NY, 3-27.

Kaul, Inge/Conceição, Pedro/Le Goulven, Katell/Mendoza, Ronald U. (Hrsg.) 2003a: Providing Global Public Goods: Managing Globalization, New York, NY.

Kaul, Inge/Conceição, Pedro/Le Goulven, Katell/Mendoza, Ronald U. 2003b: How to Improve the Provision of Global Public Goods, in: Kaul et al. 2003a, 21-58.

Kaul, Inge/Grunberg, Isabelle/Stern, Marc A. (Hrsg.) 1999: Global Public Goods: International Cooperation in the $21^{\text {st }}$ Century, New York, NY.

Kaul, Inge/Kocks, Alexander 2003: Globale Öffentliche Güter. Zur Relevanz des Begriffs, in: Brunnengräber, Achim (Hrsg.): Globale Öffentliche Güter unter Privatisierungsdruck. Festschrift für Elmar Altvater, Münster, 39-56.

Kaul, Inge/Mendoza, Ronald U. 2003: Advancing the Concept of Public Goods, in: Kaul, et al. 2003a, 78-111.

Keohane, Robert O. 1984: After Hegemony: Cooperation and Discord in the World Political Economy, Princeton, NJ.

Khanna, Jyoti/Sandler, Todd/Shimizu, Hirofumi 1998: Sharing the Financial Burden for U.N. and NATO Peacekeeping: 1976-1996, in: Journal of Conflict Resolution 42: 2, 176-195.

Khanna, Jyoti/Sandler, Todd/Shimizu, Hirofumi 1999: The Demand for UN Peacekeeping: 1975-1996, in: Kyklos 52: 3, 345-368.

Kindleberger, Chales 1981: Dominance and Leadership in the International Economy: Exploitation, Public Goods, and Free Rides, in: International Studies Quarterly 25: 2, 242-254.

Kocks, Alexander 2007: The Financing of UN Peace Operations: Providing International Public Goods in the 21st Century, Saarbrücken.

Kocks, Alexander 2009: The Politics of Humanitarian Intervention: A Public Good Approach to Peacekeeping, (Conference Paper, 2009 ISA Annual Convention »Exploring the Past, Anticipating the Future «), New York, NY.

Kölliker, Alkuin 2006: Conclusion I: Governance Arrangements and Public Good Theory: Explaining Aspects of Publicness, Inclusiveness and Delegation, in: Koenig-Archibugi, Mathias/Zürn, Michael (Hrsg.): New Modes of Governance in the Global System: Exploring Publicness, Delegation and Inclusiveness, Basingstoke, 201-235.

Lakatos, Imre 1982: Die Methodologie der wissenschaftlichen Forschungsprogramme, Wiesbaden.

Ledyard, John O. 1995: Public Goods: A Survey of Experimental Research, in: Kagel, John H./ Roth, Alvin E. (Hrsg.): The Handbook of Experimental Economics, Princeton, NJ, 111-194.

Martin, Lisa L. 1992: Interests, Power and Multilateralism, in: International Organization 46: 4, 765-792.

Martin, Lisa L. 1995: Heterogeneity, Linkage and Common Problems, in: Keohane, Robert O./ Ostrom, Elinor (Hrsg.): Local Commons and Global Interdependence: Heterogeneity and Cooperation in Two Domains, London, 71-91. 
Marx, Johannes 2006: Vielfalt oder Einheit der Theorien in den Internationalen Beziehungen. Eine systematische Rekonstruktion, Integration und Bewertung, Baden-Baden.

Mayer, Peter 2006: Macht, Gerechtigkeit und internationale Kooperation. Eine regimeanalytische Untersuchung zur internationalen Rohstoffpolitik, Baden-Baden.

Mueller, Dennis C. 2003: Public Choice III, Cambridge, MA.

North, Douglass C. 1990: Institutions, Institutional Change and Economic Performance, Cambridge, MA.

Olson, Mancur 1965: The Logic of Collective Action: Public Goods and the Theory of Groups, Cambridge, MA.

Olson, Mancur/Zeckhauser, Richard 1966: An Economic Theory of Alliances, in: The Review of Economics and Statistics 48: 1, 266-297.

Ostrom, Elinor 1990: Governing the Commons, New York, NY.

Ostrom, Elinor/Gardner, Roy/Walker, James 1994: Rules, Games, and Common-Pool Resources, Ann Arbor, MI.

Oye, Kenneth A. 1986: Explaining Cooperation under Anarchy, Princeton, NJ.

Putnam, Robert D. 1988: Diplomacy and Domestic Politics: The Logic of Two-Level Games, in: International Organization 42: 3, 427-460.

Rittberger, Volker/Kruck, Andreas/Roemund, Anne 2009: Grundzüge der Weltpolitik. Theorie und Empirie des Weltregierens, Wiesbaden.

Samuelson, Paul A. 1954: The Pure Theory of Public Expenditures, in: Review of Economics and Statistics 36: 4, 387-389.

Sandler, Todd 2002: Financing International Public Goods, in: Ferroni, Marco/Mody, Ashoka (Hrsg.): International Public Goods: Incentives, Measurement, and Financing, Norwell, MA.

Sandler, Todd 2003: Assessing the Optimal Provision of Public Goods: In Search of the Holy Grail, in: Kaul, Inge et al. 2003, 131-151.

Sandler, Todd 2004: Global Collective Action, Cambridge, MA.

Schelling, Thomas 1973: Hockey Helmets, Concealed Weapons and Daylight Savings, in: Journal of Conflict Resolution 17: 3, 381-428.

Shimizu, Hirofumi/Sandler, Todd 2002: Peacekeeping and Burden-Sharing: 1994-2000, in: Journal of Peace Research 39: 6, 651-680.

Smitha, Vincent H./Kehoea, Michael R./Cremer, Mary E. 1995: The Private Provision of Public Goods: Altruism and Voluntary Giving, in: Journal of Public Economics 58: 1, 107-126.

Snidal, Duncan 1985a: The Game Theory of International Politics, in: World Politics 38: 1, 25-57.

Snidal, Duncan 1985b: The Limits of Hegemonic Stability Theory, in: International Organization 39: 4, 579-614.

Snidal, Duncan 1995: The Politics of Scope: Endogeneous Actors, Heterogeneity and Institutions, in: Keohane, Robert O./Ostrom, Elinor (Hrsg.): Local Commons and Global Interdependence: Heterogeneity and Cooperation in Two Domains, London, 47-70.

Vicary, Simon/Sandler, Todd 2002: Weakest-Link Public Goods: Giving In-kind or Transferring Money, in: European Economic Review 46: 8, 1501-1520.

Weinmann, Joachim 2001: Wirtschaftspolitik. Allokation und kollektive Entscheidung, Berlin.

Wolf, Reinhard 2003: Zum praktischen Mehrwert aktueller Theoriebeiträge. Die Zukunft der innerwestlichen Beziehungen im Lichte konkurrierender Ansätze, in: Zeitschrift für Internationale Beziehungen 10: 1, 111-141.

Zangl, Bernhard 1994: Politik auf zwei Ebenen. Hypothesen zur Bildung internationaler Regime, in: Zeitschrift für Internationale Beziehungen 1: 2, 279-312.

Zangl, Bernhard 1999: Interessen auf zwei Ebenen: Internationale Regime in der Agrarhandels-, Währungs- und Walfangpolitik, Baden-Baden. 


\section{Literaturbericht}

Zürn, Michael 1992: Interessen und Institutionen in der internationalen Politik. Grundlegung und Anwendung des situationsstrukturellen Ansatzes, Opladen.

Zürn, Michael 1997: Assessing State Preferences and Explaining Institutional Choice: The Case of Intra-German Trade, in: International Studies Quarterly 41: 2, 295-320. 